\title{
Gut microbiota, obesity and diabetes
}

\author{
Elaine Patterson, ${ }^{1,2}$ Paul M Ryan, 2,3 John F Cryan, ${ }^{1,4}$ Timothy G Dinan, ${ }^{1,5}$ \\ R Paul Ross, ${ }^{1,6}$ Gerald F Fitzgerald, ${ }^{1,3}$ Catherine Stanton ${ }^{1,2}$
}

\begin{abstract}
${ }^{1} \mathrm{APC}$ Microbiome Institute, University College Cork, Co. Cork, Ireland

${ }^{2}$ Food Biosciences Department, Teagasc Food Research Centre, Fermoy, Co. Cork, Ireland ${ }^{3}$ School of Microbiology, University College Cork, Co. Cork, Ireland

${ }^{4}$ Department of Anatomy and Neuroscience, University College Cork, Co. Cork, Ireland ${ }^{5}$ Department of Psychiatry and Neurobehavioural Science, University College Cork, Co. Cork, Ireland

${ }^{6}$ College of Science, Engineering and Food Science, University College Cork, Co. Cork, Ireland
\end{abstract}

\section{Correspondence to} Professor Catherine Stanton, Food Biosciences Department, Teagasc Food Research Centre, Moorepark, Fermoy, Co. Cork, Ireland;

catherine.stanton@teagasc.ie

Received 19 August 2015 Revised 25 December 2015 Accepted 28 January 2016 Published Online First 24 February 2016
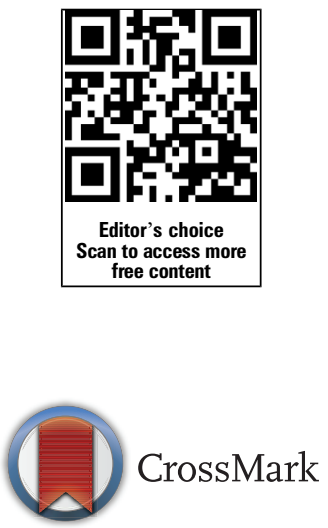

To cite: Patterson $\mathrm{E}$, Ryan PM, Cryan JF, et al. Postgrad Med J

2016:92:286-300.

\section{ABSTRACT}

The central role of the intestinal microbiota in the progression and, equally, prevention of metabolic dysfunction is becoming abundantly apparent. The symbiotic relationship between intestinal microbiota and host ensures appropriate development of the metabolic system in humans. However, disturbances in composition and, in turn, functionality of the intestinal microbiota can disrupt gut barrier function, a trip switch for metabolic endotoxemia. This low-grade chronic inflammation, brought about by the influx of inflammatory bacterial fragments into circulation through a malfunctioning gut barrier, has considerable knock-on effects for host adiposity and insulin resistance.

Conversely, recent evidence suggests that there are certain bacterial species that may interact with host metabolism through metabolite-mediated stimulation of enteric hormones and other systems outside of the gastrointestinal tract, such as the endocannabinoid system. When the abundance of these keystone species begins to decline, we see a collapse of the symbiosis, reflected in a deterioration of host metabolic health. This review will investigate the intricate axis between the microbiota and host metabolism, while also addressing the promising and novel field of probiotics as metabolic therapies.

\section{INTRODUCTION}

The Barker hypothesis, in which long-term metabolic outcomes are pre-programmed in the womb, has held centre stage for decades and may still remain at the heart of metabolic syndrome pathogenesis. ${ }^{1}{ }^{2}$ However, the gut microbiota, also referred to as the hidden organ, or the organ within an organ, harbours tens of trillions of microorganisms residing in the human intestine, which are arguably as important to metabolic health as the very organs that support them. Over the last few decades, a myriad of original research publications and comprehensive review articles make it impossible to ignore the contribution of the gut microbiota towards health and disease. It is believed that the $1-2 \mathrm{~kg}$ of microorganisms in the human gut contain $>150$-fold more genes than the human genome itself, a concept that attracts researchers from gastroenterology, physiology and microbiology and one that demolishes the notion that the gut microbiota will ever again be described as a 'forgotten organ'.

Although a very recent study has supported the possibility that the infant is first seeded with bacteria in utero from the maternal placental microbiome, ${ }^{3}$ the neonatal intestinal tract is rapidly colonised by bacteria from the mother and surrounding environment following birth. Of course, the delivery method-natural versus caesarean section-is a strong determinant of the first real microbial colonisers of the neonatal intestinal tract. ${ }^{4}$ Numerous publications have described clear differences in the gut microbiota composition between babies born via caesarean section versus natural birth ${ }^{5}{ }^{6}$ and for breast-fed versus formula-fed babies. ${ }^{7-10}$ The gut microbiota continues to develop throughout childhood and adolescence and becomes more stable as it is generally assumed that the gut microbiota after around 3 years of age closely resembles that of an adult. $^{11} 12$

The gastrointestinal (GI) tract of a healthy adult hosts around $10^{2}$ microbial cells within the highly acidic environment of the stomach, into the duodenum and jejunum. The distal ileum contains around $10^{7}-10^{8}$ microbial cells with the largest proportion of microbes finally residing in the colon, containing around $10^{11}-10^{12}$ microbial cells. Difficulties arise in culturing the microbiota from this highly anaerobic environment, with only about $10-50 \%$ successfully culturable in the laboratory. ${ }^{13}$ It is only over the last few decades with the emergence of culture-independent sequencing technologies that we have gained such a vast insight into the compositional changes that exist between individuals in both healthy and diseased states. However, while compositional studies generate a large volume of data, they fail to provide direct information regarding the microbial viability or the functional potential of the populations present and so the knowledge is somewhat limited in these aspects. ${ }^{14}$ As such, much of our understanding of the role of the gut microbiota and individual microbes comes from the use of germfree animals, that is, those born and reared without exposure to microorganisms and microbial supplementation studies. Germfree animals have highlighted the importance of a gut microbiota in influencing many aspects of host biology, including metabolism, ${ }^{15} 16$ social development ${ }^{17}$ and immunity. ${ }^{18}$ For example, germfree animals show defects in both the development of the immune system and in immune responses. They show extensive defects in the development of gut-associated lymphoid tissue and cell-turnover rates of intestinal epithelial cells and have fewer and smaller Peyer's patches and mesenteric lymph nodes compared with animals housed under specific pathogen-free (SPF) conditions. ${ }^{19-22}$ It is now fully appreciated that host-microbe interactions can together co-metabolise dietary components to produce a large array of signalling molecules that develop 'intelligent communication systems' in the body with beneficial impacts on health. 'Pharmabiotics', that is, microbially produced bioactive metabolites, such as short-chain fatty acids, conjugated fatty acids, 
exopolysaccharides and neuroactive metabolites such as $\gamma$-aminobutyric acid (GABA) and serotonin, confer health benefits on the host. ${ }^{23-25}$ Thus, host-microbe interactions are crucial for optimum health.

While culture-independent analyses have broadened our understanding of gut microbiota composition and germfree studies have helped determine the role of gut microbes in health, it is now apparent that an individual's microbial signature is not only unique but could be a predetermining factor for disease risk. Recent literature has linked gut microbiota composition with almost every disease known to man, from GI diseases, ${ }^{26-29}$ to obesity, ${ }^{30-33}$ diabetes, ${ }^{34-38}$ cancer $^{39-42}$ and even neurological and neurodegenerative disorders such as depression, ${ }^{43}$ autism, ${ }^{45-48}$ anxiety ${ }^{49} 50$ and Parkinson's disease. ${ }^{51}$ The demands for a clear link between the gut microbiota and disease have never been so high, and so advances in the development of methods used to analyse gene expression (metatranscriptomics), protein products (metaproteomics) and metabolic profiles (metabolomics) are proving crucial. ${ }^{14}$

This review will summarise the most relevant literature to date linking the gut microbiota with diet, obesity and diabetes and highlights some specific gut microbial compositional alterations that have been described as either potentially causal or protective towards metabolic disease.

\section{DIET AND OBESITY}

\section{Diet shapes the gut microbiota}

Before addressing the involvement of the gut microbiota towards shaping obesity and associated metabolic disorders, it is appropriate to first briefly discuss some recent literature that describes diet as a dominant contributing factor to gut microbial composition. Indeed, dietary factors have a profound impact on altering the gut microbiota of animals ${ }^{52-58}$ and humans. ${ }^{31}$ 59-61 This implies that health beneficial alterations to gut microbiota composition through dietary-related changes have rapidly become attractive mechanisms through which we may prevent or alleviate diseases appearing to arise from an altered microbial composition. Dietary patterns are associated with distinct combinations of bacteria in the intestine, also called enterotypes. ${ }^{62}$ Considering that the role of the gut microbiota is to ferment dietary substrates, complex diets can provide a range of growthpromoting and growth-inhibiting factors for specific phylotypes. ${ }^{63}$ The difficulty arises in deciphering which precise dietary constituents specifically promote the growth of health beneficial gut microbiota that maximise the production of pharmabiotics. ${ }^{23}$ Thus, the identification of dietary patterns or specific foods that increase bacterial diversity and promote the growth of beneficial bacteria that produce high levels of bioactive metabolites, is the ultimate research goal.

Western-style diets or various combinations of high fat (HF) diets, such as HF-high-sucrose and HF-low-plant-polysaccharide have profound effects on gut microbiota composition of animals and humans, often with conflicting results. Such HF-dietary-related gut microbial alterations often correlate with deleterious metabolic health effects. ${ }^{38} 6064$ For example, one recent study in overweight and obese human subjects demonstrated the effects of three different dietary regimens on gut microbiota composition and health outcomes. ${ }^{65}$ They found that subjects consuming what was considered to be the least healthiest dietary regimen, characterised by the high consumption of confectionary and sugary drinks and the lowest consumption of fruits, yoghurt and water, had a significantly greater inflammatory profile compared with those consuming the healthiest dietary regimen, characterised by the lowest consumption of confectionary and sugary drinks and the highest consumption of fruits, yoghurts and soups. ${ }^{65}$ Interestingly, although the data did not highlight any significant changes to the seven gut microbial groups studied between the groups, the healthiest dietary regimen was associated with the highest microbial gene cluster or the highest microbial diversity/richness. ${ }^{65}$ This is indeed a significant finding as the importance of microbial diversity has previously been identified as a significant factor influenced by diet and exercise ${ }^{66}$ and which is also decreased during overweight and obese disease states. ${ }^{67}$ Fat type is also a strong determinant of inflammation and gut microbiota composition. Diets rich in saturated fat (lard) are associated with increased white adipose tissue (WAT) inflammation and metabolic disease, while diets rich in polyunsaturated fatty acids (fish oil) can counteract inflammation to promote a lean and metabolically healthy phenotype. ${ }^{68}{ }^{69}$ In fact, mice fed fish oil have increased levels of taxa from the genera Lactobacillus and Akkermansia (discussed in more detail later) while mice fed lard have increased levels of taxa related to Bilophila. ${ }^{69}$ Bilophila wadsworthia has previously been shown to exacerbate colitis in genetically susceptible models. ${ }^{70}$ While previous studies have shown that microbial factors can directly contribute to WAT inflammation through Toll-like receptor (TLR) signalling, ${ }^{71} 72$ dietary and host factors are also strong TLR ligands and so whether gut microbial factors initiate TLR signalling with an effect on health is uncertain. Caesar et al proved that lard-induced WAT inflammation is mediated through gut microbial activation of TLR4. Trif-/- and Myd88-/- (adaptor proteins necessary for interpreting TLR signalling) mice were protected against lard-induced WAT inflammation and impaired insulin sensitivity. Furthermore, the authors discovered that serum lipopolysaccharide (LPS) levels were higher in mice fed lard compared with those fed fish oil, indicating that microbial factors are present in the periphery that affect WAT inflammation. The impact of gut microbial factors on lard-induced WAT inflammation was further elucidated by comparing the effects of lard and fish oil in conventionally raised and germfree mice. These results demonstrated an adiposity-independent link between the gut microbiota and WAT inflammation, which led the authors to conclude that microbially derived products act as mediators of inflammation through TLR signalling. This highlights the gut microbiota as independent factors aggravating inflammation following saturated fat feeding. ${ }^{69}$

The data of Clarke et $a l^{66}$ demonstrate the importance of dietary variation and protein consumption, coupled with exercise, towards shaping gut microbial composition. In this study, protein consumption positively correlated with high microbial diversity (22 distinct phyla) and athletes with a low body mass index had significantly higher levels of Akkermansia, previously shown to inversely correlate with obesity in both mice and humans. $^{73}$ These results highlight the importance of dietary macronutrients and exercise on positively shaping the gut microbiota composition. Fascinating discoveries have been made from the recently development multi-taxon Insertion Sequencing (INSeq) method, which monitors the genetic factors that enable members of the gut microbial community to flourish within this niche. ${ }^{75}$ This approach can be used to understand the mechanisms (gene-level characterisation) that determine gut microbial fitness and perturbation through diet, disease and clinical treatment. Using this approach, Wu et $a l^{75}$ revealed a strain-specific and diet-specific fitness determinant (arbinoxylan utilisation locus) in Bacteroides cellulosilyticus WH2 that is critical for the organism's fitness during HF/simple-sugar feeding. What's more, supplementation of drinking water with arbinoxylan 
(cereal-derived hemicellulose) in mice consuming this diet selectively increased the abundance of B. cellulosilyticus. ${ }^{75}$ Thus, by increasing our understanding of how host genotypic and phenotypic factors affect gut microbial niches, multi-taxon INSeq can provide gene-level characterisation of species and strain-specific fitness determinants that enable therapeutic interventions (eg, prebiotic supplementation) to manipulate gut microbial community structure and function in health.

It is, however, often very difficult to determine the extent by which dietary factors affect gut microbiota composition, aside from genetic factors. While we appreciate that the component members of the gut microbiota can be stable for years, ${ }^{76}$ the community structure or the relative abundances of each member is highly dynamic. ${ }^{77}$ To this end, Carmody et $a l^{78}$ described the role of host genetics in driving dietary-related alterations in gut microbiota composition. This study proved that diet is a predominant environmental factor over host genetics in shaping gut microbiota composition. This theory was based on previous studies whereby monozygotic twins failed to possess more similar gut microbiota profiles when compared with dizygotic twins at various ages from infancy to adulthood, suggesting that host genetic factors play a minor role in altering the human gut microbiota. ${ }^{30} 79$ The results showed that consumption of a HF-high-sugar diet reproducibly altered the gut microbiota in five inbred mouse strains, mice deficient for genes relevant to host-microbial interactions $\left(\mathrm{MyD} 88^{-/-}, \mathrm{NOD} 2^{-/-}, o b / o b\right.$ and $\operatorname{Rag} 1^{-/-}$) and in $>200$ outbred strains. ${ }^{78}$ Thus, the study concluded that diet is a dominant environmental factor that alters the gut microbial community universally in the host, despite genetic variation. Furthermore, dietary-related gut microbial changes occurred within an average of 3.5 days and were reversible. ${ }^{78}$ Indeed, the interactions between host genetics and environmental conditions have recently been described as confounding factors in the development of metabolic syndrome. Ussar et $\mathrm{al}^{80}$ discovered that the phenotype of the 129SvEv/Tac (129T) mouse strain from Taconic Farms, usually susceptible to developing diet-induced obesity and enlarged livers on HF-diet but with normal insulin sensitivity and mild glucose intolerance, could be altered through environmental normalisation over at least three generations to become 'metabolic syndrome resistant', similar to the phenotype of the genetically related $129 \mathrm{SvEv} / \mathrm{ImJ}$ (129J) strain from Jackson Laboratories. Environmental normalisation of the 129T strain remodelled the microbiota, and these changes correlated with a metabolic resistant phenotype. ${ }^{80}$ It must be noted that environmental normalisation of the $\mathrm{C} 57 \mathrm{Bl} / 6 \mathrm{~J}$ and the $129 \mathrm{~J}$ strains had no significant effect on metabolic phenotype, despite a changed gut microbiota. ${ }^{80}$ This study is crucial in describing the importance of dietary, genetic and microbial interactions on the development of metabolic syndrome.

If diet is a dominant environmental factor driving gut microbial changes, then Bolnick et $a l^{81}$ have demonstrated that these changes are sex dependent. Laboratory fish, laboratory mice and humans have all demonstrated sex-dependent, diet-microbiota associations. Interestingly, this means that diet affects gut microbiota differently in males and females. From a clinical perspective, if the intention is to take advantage of the dominant effect dietary factors have on shaping a more healthful gut microbiota, perhaps future therapies ought to be sex specific.

\section{The gut microbiota shape obesity}

On a global scale, projection estimations have predicted that the obesity epidemic rose from 400 million obese adults in 2005 to $>700$ million in 2015, a trend that is set to continue towards
$2030 .^{82} 83$ Characterised by an excess accumulation of body fat and a low-grade systemic inflammatory tone, behavioural patterns including dietary trends remain the underlying cause of this imbalance of energy input versus expenditure. Convenient, easily accessible, energy-dense foods and overnutrition represent major starting points altering lipid metabolism, systemic inflammation and the gut microbiota. Although studies highlighting the impact of dietary patterns and overnutrition on gut microbiota composition are plentiful, few decipher a clear link between the microbial changes observed and the mechanisms involved. In addition, the definition of this 'microbial signature' of obesity is ever changing, each new study describing conflicting compositional results to the last. Nonetheless, microbial intervention or the promotion/inhibition of the growth of certain species are attractive proposals for the future prevention of this obesity epidemic.

Turnbaugh et $a l^{84}$ performed one of the first studies that definitively linked the gut microbiota with weight gain, caused by an increase in the energy-harvesting capabilities of the 'obesemicrobiota'. The gut microbiome possesses glycoside hydrolase enzymes, not found in the human genome and that are crucially involved in hydrolysing and fermenting a wide variety of dietary polysaccharides that enhance host energy status. ${ }^{85} 86$ The microbially derived energy from these otherwise indigestible complex polysaccharides are in the form of short-chain fatty acids (SCFA) (acetate, butyrate and propionate, predominantly). SCFAs are crucial to host health and are the principal energy source for colonocytes as well as playing a key role in the prevention and treatment of metabolic and bowel disorders and certain types of cancer. ${ }^{87-91}$ Germfree mice develop less body fat than conventionally raised mice and germfree mice colonised with the gut microbiota of conventional mice, despite increasing their food intake and decreasing their metabolic rate. ${ }^{92}$ Furthermore, germfree mice colonised with an 'obese-microbiota' isolated from genetically obese $o b / o b$ mice had a greater percentage increase in body fat than those colonised with a 'leanmicrobiota', despite no significant differences in energy consumption or initial body fat/weight between the groups. ${ }^{84}$ In this setting, the germfree mouse model has provided invaluable insights into the link between gut microbiota and body fat accumulation in the host.

At the compositional level, obesity is associated with changes in abundance ratios of two of the most dominant phyla (constituting $>90 \%$ of known phylogenetic categories), namely Firmicutes and Bacteroidetes (figure 1). However, while some studies have described increases in the proportion of Firmicutes to Bacteroidetes in the obese phenotype, compared with normal weight individuals ${ }^{30}$ and weight loss is said to reduce the Firmicutes to Bacteroidetes ratio in human subjects, ${ }^{31}$ these findings are not universal ${ }^{32} 53$ and so the usefulness of the Firmicutes:Bacteroidetes ratio as a compositional biomarker for obesity remains unclear.

The low-grade inflammatory state characteristic of obesity can be further exacerbated by microbiota-associated inflammatory processes (figure 1). Cani et $a l^{7193}$ first described the concept of metabolic endotoxemia (ie, increased plasma LPS) in a series of experiments in mice. The first finding was that both fat feeding and a HF diet increased plasma LPS levels by two-fold and five-fold, respectively. ${ }^{71}$ It was also observed that subcutaneous infusions of LPS into mice increased insulin resistance and obesity similar to that seen after feeding the HF diet. ${ }^{71}$ This positive correlation between HF feeding, genetic obesity $(o b / o b)$, type 2 diabetes (T2D) $(d b / d b)$ and metabolic endotoxemia has since been confirmed in mice. ${ }^{94} 95$ Furthermore, 


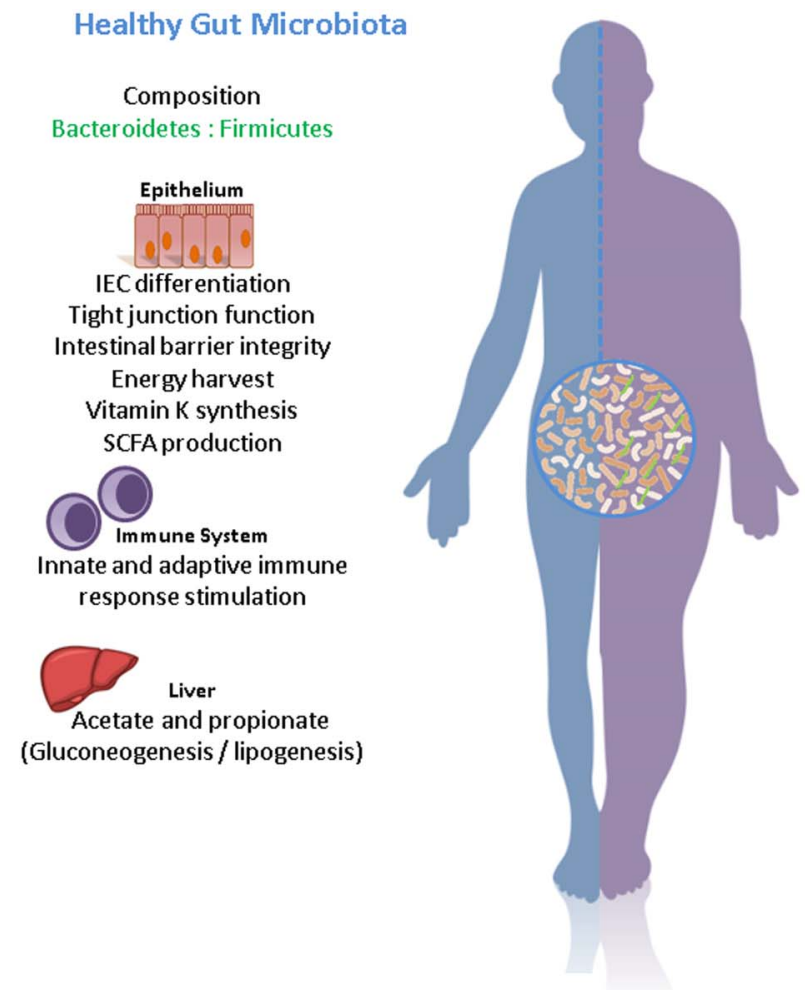

Obese-Diabetic Microbiota

Composition

Bacteroidetes : Firmicutes

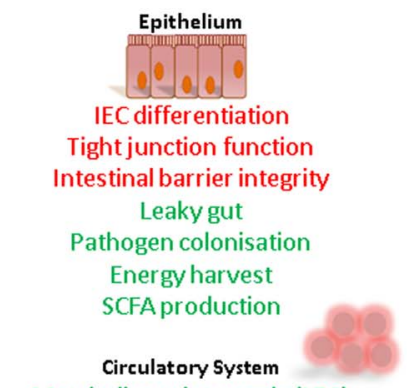

Metabolic endotoxemia (LPS)

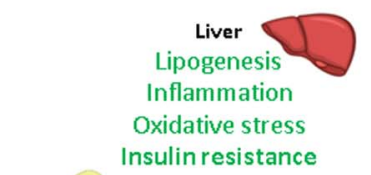

ulin resistance

Adipose Tissue

Inflammation

Oxidative stress

Macrophage infiltration

Insulin resistance

Figure 1 Compositional and functional alterations in the healthy gut microbiota versus the obese-diabetic microbiota. The metabolic processes in peripheral organs leading to increased adiposity, inflammation, oxidative stress, insulin resistance and lipogenesis are associated with the altered microbiota profile associated with the obese-diabetic phenotype. IEC, intestinal epithelial cell; LPS, lipopolysaccharide; SCFA, short chain fatty acid.

probiotic, ${ }^{93}$ prebiotic $^{96}$ and antibiotic ${ }^{94}$ therapies have improved metabolic inflammatory parameters in HF diet fed and ob/ob mice. In a healthy state, the translocation of LPS, through the intestinal epithelium, is restricted as it damages the integrity of the intestinal barrier. This gut barrier dysfunction observed in the obese state may represent an open door for microbes and microbial-derived LPS endotoxin to enter systemic circulation, increasing gut permeability ${ }^{94-99}$ and paracellular permeability by disrupting tight-junction proteins that link epithelial cells together (eg, claudin, occludin and zonula occludens). ${ }^{100}$ Mechanistically, increased gut permeability in obese mice may have been associated with an alteration in the expression, localisation and distribution of two tight-junction proteins (occludin and zonula occludens 1) in the small intestine. ${ }^{94} 959799$ Thus, microbial-associated metabolic endotoxemia appears to represent one of the most important links between the gut microbiota, intestinal permeability and the low-grade inflammatory state linked with obesity.

Ridaura $e t \mathrm{al}^{101}$ recently described a fascinating link between diet, gut microbial composition and obesity. Their cohort of female twin pairs, discordant for obesity, provided a unique opportunity to examine the interrelations between obesity, associated metabolic disorders, diet and the gut microbiota. Transplantation of human gut microbiota from each member of discordant twin pairs for obesity permitted the donors' communities to be replicated in germfree mice, that is, transplantation of a human 'obese-microbiota' from one co-twin transmitted an increase in total body and fat mass, as well as other obesity-associated metabolic phenotypes with corresponding faecal bacterial cultures in germfree mice. ${ }^{101}$ Furthermore, cohousing mice transplanted with this human 'obese-microbiota' with mice transplanted with a human 'lean-microbiota' prevented the development of obesity. ${ }^{101}$ Invasion of specific members of the Bacteroidetes phylum from the transplanted mice with the 'lean-microbiota' to their 'obese-microbiota' littermates correlated with the prevention of obesity development. ${ }^{101}$ In addition, following consumption of a low-fat, high-fibre diet, the 'obese-microbiota' failed to colonise lean mice as efficiently as it had in mice consuming a HF, low-fibre diet when cohoused with mice transplanted with human 'lean-microbiota'. ${ }^{101}$ The results of this study highlight both diet and environment as crucial factors involved in altering the microbiota to affect the metabolic phenotype in the host.

Compositional differences are evident in the obese relative to the lean state. ${ }^{67}$ With obese individuals harbouring lower bacterial richness and characterised by greater adiposity, with increased insulin resistance, dyslipidemia and higher rates of systemic inflammation (increased $\mathrm{C}$ reactive protein), when compared with high bacterial richness individuals. ${ }^{67}$ Importantly, the significant differences in bacterial richness and thus microbial communities/metabolic capabilities between obese and lean groups in this study were based on 46 genera. Bacteroides, Parabacteroides, Ruminococcus, Campylobacter, Dialister, Porphyromonas, Staphylococcus and Anaerostipes were more dominant in subjects of low bacterial richness with an obese phenotype, while Faecalibacterium, Bifidobacterium, Lactobacillus, Butyrivibrio, Alistipes, Akkermansia, Coprococcus and Methanobrevibacter were more prevalent in subjects with high bacterial richness and a lean phenotype. ${ }^{67}$ These compositional data permitted a closer look to be taken at the microbial metabolic capabilities of individuals with high and low bacterial richness. It was concluded from the vast amount of data that subjects with low bacterial richness and an obese phenotype had (1) a reduction in butyrate-producing bacteria (2), a reduction in 
hydrogen and methane production (3), an increase in mucus degradation and (4) an increase in the potential to manage oxidative stress. ${ }^{67}$ Thus, it appears that obese individuals have lower bacterial richness and harbour a microbiota that predisposes them to further inflammation.

It is clear that obesity is associated with an altered gut microbiota that worsens this debilitating disease. Diversity is key to increasing the metabolic capabilities of the gut microbiota in order to prevent worsening metabolic endotoxemia, mucus degradation and oxidative stress. In the vast gut microbial ecosystem, two residents have been the subject of extensive research over the last number of years due to their negative association with the obese phenotype. Intriguing data have supported an antiobese movement of these two species, so much so that alleviating obesity and associated metabolic disorders through increasing their abundances has become a real opportunity.

\section{Akkermansia muciniphila}

A. muciniphila is a mucin-degrading Gram-negative bacterium residing in the mucus layer of intestinal epithelial cells and that constitutes about $3-5 \%$ of the gut microbiota biomass in humans. ${ }^{102}$ Murine $^{73}$ and human ${ }^{74}$ studies have demonstrated an inverse correlation between A. muciniphila and overweight, obesity and diabetes. Everard et $a l^{7399}$ reported convincing data describing a potential protective function of A. muciniphila in murine models of obesity. They describe a comprehensive data set highlighting an inverse correlation between genetic and dietary models of obesity and gut concentrations of A. muciniphila. ${ }^{73}$ Furthermore, prebiotic supplementation with oligofructose (dietary fibre) dramatically increased the abundance of $A$. muciniphila in genetically obese mice ${ }^{99}$ and restored the decreased basal levels of $A$. muciniphila associated with obesity, improving metabolic function and endotoxemia. ${ }^{73}$ Such studies suggest that $A$. muciniphila plays a role in controlling fat storage, adipose tissue inflammation and glucose metabolism.

A recent ex vivo model based on mouse ileal organoids has provided some novel data linking the gut microbiota and the host. ${ }^{103}$ Lukovac et al ${ }^{103}$ reported that ileal organoid expression of fasting induced adipocyte factor, G-protein coupled receptor (Gpr)-43, histone deacetylases and peroxisome proliferatoractivated receptor- $\gamma$ were modulated by $A$. muciniphila and the SCFA, propionate. ${ }^{104}$ Thus, it is clear that A. muciniphila and its SCFA regulate transcription factors and genes involved in cell cycle control, lipolysis and satiety. ${ }^{103}$ Previously, the effect of A. muciniphila on cell survival, more specifically in cell death receptor signalling within ileal tissue, has also been described in vivo in germfree mice. ${ }^{105}$

Early life therapy in the non-obese diabetic (NOD) mouse model of diabetes with antibiotics increased the relative abundance of A. muciniphila with an improvement to the diabetic phenotype. ${ }^{106}$ In addition, Dubourg et $a l^{107}$ demonstrated a dramatic colonisation of the human gut microbiota by the phylum Verrucomicrobia following broad-spectrum antibiotic treatment. Interestingly, all Verrucomicrobia phylotypes were represented by A. muciniphila. ${ }^{107}$ This shift in gut microbial composition highlights the susceptibility of some bacterial populations following antibiotic therapy and the susceptibility of other more resistant microorganisms to thrive under antibiotic pressure. ${ }^{108}$ In a follow-up study, Caputo et al ${ }^{109}$ assembled the whole genomes of A. muciniphila isolated directly from the stool of the patients who partook in the previous study using a metagenomic approach.

Despite the confounding evidence suggesting the antiinflammatory effects of $A$. muciniphila in obesity, ${ }^{73}$ diabetes ${ }^{106}$ and colitis, ${ }^{110}$ others have failed to demonstrate such health benefits. Higher abundances of A. muciniphila have been observed in healthy controls in some human gut microbiota studies. ${ }^{38}$ In addition, the Chinese MGWAS study reported that 337 of the 2176 A. muciniphila-related genes were more abundant in T2D patients compared with healthy controls. ${ }^{37}$ It has also been suggested that $A$. muciniphila could facilitate intestinal inflammation through mucin degradation as previously shown in Salmonella typhimurium-infected mice. ${ }^{111}$ Such conflicting results highlight the need for further studies to decipher the precise role of $A$. muciniphila in inflammatory-related diseases and to clarify previous discrepancies.

\section{Faecalibacterium prausnitzii}

Faecalibacterium prausnitzii is also an abundant intestinal microbe comprising approximately $4 \%$ of the mainly luminal microbiota. $^{105}$ Patients with inflammatory symptoms of inflammatory bowel disease (IBD), obesity and related metabolic dysfunctions harbour reduced abundances of F. prausnitzii. ${ }^{37} 3874112-114$ Some recent functional studies have described increased F. prausnitzii in overweight subjects who underwent a fasting programme over 1 week followed by supplementation of a probiotic formula for 6 weeks. ${ }^{114}$ Dietary supplementation with a type 3-resistant starch in the SATIN project altered the gut microbiota composition in overweight human subjects by enriching taxa involved in starch degradation (Ruminococcus bromii) and butyrate production (Eubacterium rectale) and increasing $F$. prausnitzii. ${ }^{115}$ This study describes the association between microbial fermentation of resistant starch and satiety. ${ }^{115}$ Furthermore, F. prausnitzii has shown protective effects in both acute ${ }^{112}$ and chronic ${ }^{116}$ inflammatory responses in chemically induced models of inflammation. Considering the low-grade inflammatory tone, symptomatic of IBD and often associated with obesity, Martin et al recently investigated the protective effects of $F$. prausnitzii in a preclinical murine model of colitis. ${ }^{117}$ Importantly, decreased intestinal permeability was a feature following F. prausnitzii supplementation in mice chemically subjected to chronic low-grade inflammation and gut dysfunction compared with controls. ${ }^{117}$ Results from this study prompted the authors to describe $F$. prausnitzii as a potential novel probiotic that can be successfully used in the treatment of gut dysfunction and inflammation. Although the authors propose that the protective effects of $F$. prausnitzii could at least be in part due to the enhancement of tight junction proteins in the epithelial cells, promoting barrier function, further studies are needed to determine the precise role of $F$. prausnitzii in obesity and inflammatory-related metabolic diseases.

\section{The endocannabinoid system and obesity}

The endocannabinoid (eCB) system has been proposed to be involved in the regulation of energy homeostasis, appetite ${ }^{118}$ and gut barrier function ${ }^{119}$ via the microbiota-gut-brain axis during obesity. ${ }^{120}$ The eCB system is composed mainly of the bioactive lipids anandamide (AEA; an $\mathrm{N}$-arachidonoylethanolamine), 2-arachidonoylgycerol (2-AG) (synthesised locally in the GI tract), the proteins that regulate their production/degradation and the cannabinoid receptors $\mathrm{CB}_{1}$ and $\mathrm{CB}_{2}$, through which they signal. While both AEA and 2-AG are $C_{1}$ and $C_{2}$ ligands, $C_{1}$ is expressed mainly in the liver, pancreas, adipose tissue and the peripheral and central nervous systems. ${ }^{121} \mathrm{CB}_{2}$ is expressed mainly in the immune cells, in addition to the brain, pancreas and adipose tissue. ${ }^{122} \mathrm{In}$ short, $\mathrm{CB}_{1}$ activation reduces gastric motility while $\mathrm{CB}_{2}$ activation reduces an inflammatory response. Interestingly, obesity is 
associated with an increase in eCB system tone $\mathrm{e}^{123}$ and an altered expression of $\mathrm{CB}_{1}$. For example, $\mathrm{CB}_{1}$ receptor knockout mice have upregulated $\mathrm{eCB}$ signalling in a diet-induced obese mouse model. ${ }^{124}$ Muccioli et al $^{125}$ have shown in genetically obese and T2D mice that $\mathrm{CB}_{1}$ receptor antagonists decreased gut permeability. Furthermore, by altering gut microbiota composition through prebiotic feeding, colonic $\mathrm{CB}_{1}$ mRNA expression is reduced and antibiotic treatment also decreased the expression of the $\mathrm{CB}_{1}$ receptor in the colon. ${ }^{125}$ These results correlate with a reduction in colonic AEA (endogenous $\mathrm{CB}_{1}$ ligand), an increase in fatty acid amine hydrolase (the main enzyme in degradation of AEA) and reduced plasma LPS levels. ${ }^{125}$ This suggests that the gut microbiota indeed play a role in regulating obesity and obesity-associated metabolic endotoxemia through the $\mathrm{eCB}$ system.

\section{The gut microbiota and type 1 diabetes}

Unlike T2D, type 1 diabetes (T1D) is primarily caused by a genetic disposition to pancreatic $\beta$-cell autoimmunity, ${ }^{126}$ in which inflammatory $\mathrm{T}$ cells are acknowledged to play a central role. ${ }^{127}$ Among the genetic loci identified in T1D patients, it is specific human leucocyte antigen (HLA) genotypes that have the strongest identified genetic factor to date. ${ }^{128} 129$ However, twin and family studies have shown that only a fraction of genetically predisposed individuals will go on to develop T1D, ${ }^{130-132}$ and with the incidence of T1D increasing steadily by about 3-4\% over the past few decades, particularly among young children in developed countries, ${ }^{133}$ environmental factors seemingly play a stronger role than once anticipated towards triggering this autoimmune response. The impact of environmental factors on the increasing incidence and decreasing age of diagnosis of children with lower-risk class II HLA genes have been described. ${ }^{134}$ Infant feeding practices, diet and viruses are all relevant environmental triggers shown to play a role in disease onset. Moreover, risk of T1D onset in childhood is higher in children delivered by caesarean section, ${ }^{135}$ where there is also an altered gut microbiota composition. ${ }^{4}$

Genetically induced, that is, the NOD mouse and the biobreeding diabetes prone (BBDP)/diabetes-resistant (BBDR) and chemically induced, that is, streptozotocin (STZ) and alloxan-induced T1D animal models all describe convincing data linking an altered microbiota with disease. Wen et al ${ }^{136}$ previously described the importance of the gut microbiota and its interactions with the host innate immune system in modulating T1D onset. MyD88 is an adaptor for multiple innate immune receptors, including TLRs involved in microbial sensing. It is the MyD88 signalling pathway that is required for autoimmune diabetes development in NOD mice under SPF conditions. $^{136}$ The knockout of MyD88 in NOD mice completely protects against T1D development. ${ }^{136}$ Furthermore, heterozygous MyD88-/- NOD mice, which normally develop robust diabetes, are protected from disease development when exposed from birth to the gut microbiota of a MyD88 knockout NOD donor. ${ }^{136}$ Thus, disease progression in the NOD mouse is partly driven by an exaggerated innate immune response to symbiotic microbiota and altering microbial composition can curtail this response and prevent disease. Exposure to bacterial antigens and infections has also been shown to decrease the risk of NOD mice developing T1D. ${ }^{137}$

Further evidence for the role of the gut microbiota in T1D arises from the studies of BBDP and BBDR rodents. Interestingly, treating BBDP mice with antibiotics decreased their risk of developing T1D. ${ }^{138}$ Focusing on the proportions of bacteria found in BBDP rats at disease onset, probiotic-like microbiota such as bifidobacteria and lactobacilli were lower while Bacteroides, Ruminococcus and Eubacterium were higher compared with BBDR rats. ${ }^{139}$ However, pre-T1D onset the gut microbiota of BBDP rats was significantly different to that of BBDR rats ${ }^{139}$ and so it is difficult to conclude from this study whether microbial alterations were causal or consequential of disease since changes were observed before and after disease onset. Recently, we described distinct microbial alterations in STZ-induced T1D rats over time. ${ }^{140}$ In this study, rats were injected with a single dose of the pancreatic $\beta$-cell toxin STZ and T1D was apparent following 1 week. Although this model was severe, given the lack of insulin therapy throughout the 5 weeks of disease, with blood glucose levels between 500 and $600 \mathrm{mg} / \mathrm{dL}$ in diabetic rats, it does provide a comprehensive account of the microbial alterations that occurred following disease onset and progression over time. ${ }^{140}$ Most notably, T1D onset was associated with a shift in the Bacteroidetes: Firmicutes ratio, while at the genus level increased proportions of lactic acid producing, Bifidobacterium and Lactobacillus were identified at the later stages of T1D progression. Coincidently, T1D increased caecal lactate levels and decreased caecal butyrate levels compared with healthy controls. It has been suggested that the imbalance of bacteria that produce SCFA can affect gut permeability and cause T1D. Brown et $a l^{35}$ have described the importance of butyrate in intestinal permeability during T1D. Heightened gut permeability has been demonstrated as one of the phenomena that precedes the clinical onset of T1D in both animal models of autoimmune diabetes as well as in patients with T1D and pre-diabetic individuals. ${ }^{141-145}$ It is the fate of lactate that is crucial in determining intestinal health as conversion to butyrate results in mucin synthesis ${ }^{146-149}$ and tighter junctions ${ }^{150} 151$ while conversion to other SCFA such as acetate and propionate does not induce mucin synthesis. ${ }^{35}$ In addition, butyrate contributes to colonic health through its anti-inflammatory properties ${ }^{152-154}$ and decreases bacterial transport across metabolically stressed epithelial cells, ${ }^{153}$ thus preventing the development of the so-called 'leaky gut'. Furthermore, the STZ-induced T1D rats demonstrated a reduced microbial diversity after just 1 week of disease onset that did not recover over the duration of the trial. ${ }^{140}$ Previously described are the links between a reduced microbial diversity and obesity-associated metabolic diseases. ${ }^{67}$

The altered gut microbiota associated with T1D has also been reported in numerous human studies. A 4-matched case-control study in Finland reported that the gut microbiota differed between children who were healthy and those with autoimmune disorders. ${ }^{155} \mathrm{~A}$ higher level of Bacteroidetes relative to Firmicutes approximately 6 months after birth was observed in those who eventually developed T1D and suggested that this ratio of Bacteroidetes to Firmicutes increased over time in autoimmune cases, but declined in those who did not develop T1D. ${ }^{155}$ The authors concluded that the ratio of Bacteroidetes to Firmicutes could be an early diagnostic marker of pending autoimmunity problems. Such case-control studies have associated a higher Bacteroides abundance with autoimmunity, with increased Bacteroides ovatus and Bacteroides uniformis and decreased Bacteroides fragilis in T1D patients. ${ }^{34-36} 155$ Furthermore, butyrate producers such as Faecalibacterium and Roseburia and mucin degraders Prevotella and Akkermansia were all found in greater proportions in healthy controls compared with cases and have been described as protective against T1D. ${ }^{34-36155}$ A recent German study, however, found no differences in the Bacteroides abundance between children who developed anti-islet cell autoimmunity and healthy controls between 6 months of age and 3 years. ${ }^{156}$ They did, however, 
observe an increase in the abundance of the phylum Verrucomicrobia, of which Akkermansia is the only known gut microbial member. ${ }^{105} 156$ Previously, Brown et al ${ }^{35}$ suggested that a gut microbiota abundant with butyrate producers, increases mucin production, increases tight junction assembly, and thus, increases epithelial cell integrity. Therefore, with increased mucin production, a favourable niche exists for mucin degraders such as A. muciniphila that could possibly be used as an indicator of gut integrity. ${ }^{35}$ A very recent study has extensively examined the gut microbiota composition and diversity of 33 infants genetically predisposed to T1D. ${ }^{157}$ The authors acknowledged the limitations of the study being that all children in their cohort carry T1D-predisposing HLA alleles and are restricted to the countries of Finland and Estonia; nonetheless, their results are extremely novel in linking the gut microbiota with disease. ${ }^{157}$ The study reported a $25 \%$ reduction in gut microbial diversity in T1D patients compared with seroconverters (those positive for at least two of the five autoantibodies analysed; insulin autoantibodies, glutamic acid decarboxylase antibodies, islet antigen-2 antibodies, zince transporter eight antibodies and islet cell antibodies) and non-converters. Importantly, this was associated with alterations to both phylogenetic microbial composition and metabolic pathways in T1D diagnosed children throughout the study period. ${ }^{157}$ In addition, these shifts occurred prior to disease onset but after seroconversion and were specific to T1D progressors but not seen in seroconverters without disease. ${ }^{157}$ Remarkably, this novel data set describes a gut microbial profile that precedes T1D development but that is not found in seroconverters without disease or non-converters. Such results highlight a unique opportunity to exploit gut microbial profiles in distinguishing between T1D progressors from non-progressors and so has major implications for the future treatment of disease, based on microbial profiles.

\section{The gut microbiota and T2D}

It is estimated that $>80 \%$ of patients with T2D are overweight and increased body weight is now recognised as the greatest risk factor of T2D, aside from genetic and lifestyle factors. Numerous studies have focused on the relationship between obesity and the gut microbiota; however, with T2D now generally considered an attribute to obesity, observational studies linking glucose intolerance and insulin resistance associated with T2D with the gut microbiota are somewhat lacking. Once again, inflammatory pathways underscore the evolution of insulin resistance. ${ }^{158}$ Patients with metabolic syndrome and T2D exhibit a remarkable endotoxemia. ${ }^{159} 160$ Recent studies have suggested that LPS may be crucially involved in T2D-associated inflammation. Serino et al ${ }^{161}$ reported an increase in gut permeability and endotoxemia with a specific gut microbial profile in mice receiving a HF diet until they became diabetic. In line with this, Amar et al ${ }^{162} 163$ described the concept of 'metabolic infection' to define the contribution of the gut microbiota in the endotoxemia-associated inflammation coupled with insulin resistance in T2D. Blood levels of certain bacterial DNA $(>85 \%$ derived from Proteobacteria) were found to be enhanced in prediabetes. ${ }^{163}$ Microbially derived endotoxin could, therefore, play a significant role in insulin resistance associated with T2D.

Qin et $a^{37}$ were among the first authors to complete a significant metagenome-wide association study in T2D. High-throughput sequencing on a cohort of Chinese patients with T2D demonstrated some interesting correlations between the clinical data and gut microbial composition. ${ }^{37}$ While butyrate-producing bacteria (Clostridiales sp. SS3/4, E. rectale, F. prausnitzii, Roseburia intestinalis, among others) were enriched in healthy control subjects, patients with T2D exhibited an altered gut microbial profile, marked by decreased butyrate-producing bacteria such as $R$. intestinalis and F. prausnitzii. ${ }^{37}$ Interestingly, the T2D gut microbial profile was colonised by opportunistic pathogens such as Bacteroides caccae, various Clostridiales, Escherichia coli and the sulfate-reducing species Desulfovibrio. ${ }^{37}$ The functional capacity of the T2D gut microbial profile was linked with enrichment in the membrane transport of sugars, oxidative stress responses, branched chain amino acid transport, sulfate reduction and decreased butyrate biosynthesis. ${ }^{37}$ In total, $>3 \%$ of the gut microbial genes differed between healthy controls and patients with T2D. ${ }^{37}$

In a follow-up study, Karlsson $e t \mathrm{al}^{74}$ reported compositional changes linked with T2D in a Scandinavian cohort of postmenopausal women. The data presented in this cohort again revealed significant decreases in butyrate producers $R$. intestinalis and $F$. prausnitzii in T2D women compared with women with impaired glucose tolerance. ${ }^{74}$ Both cohorts also demonstrated increases in Lactobacillus species associated with T2D. ${ }^{37}{ }^{74}$ As already discussed, butyrate plays a significant role in the maintenance of intestinal epithelial cell integrity with important functions in the prevention of 'leaky gut' associated with diabetes. Therefore, the role of SCFA, particularly butyrate and butyrate-producing bacteria, is crucial for health in obesity and diabetes. As studies in T2D consistently reveal that production of SCFAs, especially butyrate, is impaired, it is reasonable to assume that such mechanisms may contribute to the low-grade inflammation observed in such disorders. ${ }^{164}$

Although plentiful in compositional data, both Chinese and Scandinavian cohorts lacked data determining whether T2D-specific medication may or may not have influenced gut microbial profiles of patients or whether such medications could have 'corrected' the altered microbial ecosystem associated with T2D. Recent promising data described a role for metformin, one of the most widely prescribed T2D therapeutic agents, towards improving the gut microbial profile in T2D. ${ }^{165}$ Following 6 weeks of metformin treatment in HF-diet fed mice, both the glycaemic profile and microbial profile (29 genera) was altered, when compared with HF-fed controls. ${ }^{165}$ Interestingly, HF-fed mice treated with metformin showed higher abundances of the mucin-degrading bacterium Akkermansia that correlated with increased mucin-producing goblet cells. ${ }^{165}$ Furthermore, oral administration of A. muciniphila to HF-diet fed mice in the absence of metformin, significantly enhanced glucose tolerance and attenuated adipose tissue inflammation. ${ }^{165}$ This improvement in glucose tolerance associated with A. muciniphila had also previously been identified. ${ }^{73}$ The results of Shin et al were further confirmed a few months later by Lee and Ko, ${ }^{166}$ who demonstrated an increase in A. muciniphila following metformin treatment with a negative correlation between glycaemia and A. muciniphila abundance. In line with this, Zhang et $a l^{38}$ reported that the abundances of Verrucomicrobiaceae and $A$. muciniphila were significantly reduced in patients with prediabetes compared with healthy controls. Thus, A. muciniphila may contribute to the antidiabetic effect of metformin, suggesting that pharmacological manipulation of the gut microbiota in favour of Akkermansia may be a potential treatment for T2D. These studies describe attractive data sets that highlight the possibility of gut microbial manipulation to alleviate T2D and describes a new mechanism for the therapeutic effect of metformin in treating T2D.

Following on from this, one recent study dissected 784 available human gut genomes from the MetaHIT project, novel Danish MetaHIT genomes, cohorts of female Swedish patients 
with T2D and Chinese patients with T2D to demonstrate how metformin impacted on gut microbial profiles. ${ }^{167}$ For these 784 gut metagenomes, taxonomic and functional profiles were determined. Using the available data sets and controlling for metformin, the authors identified a unified signature of gut microbiome shifts in $\mathrm{T} 2 \mathrm{D}$, characterised by a depletion in butyrate-producing taxa. ${ }^{167}$ It was shown that gut microbial function was altered following metformin treatment such that intestinal lipid absorption and LPS-triggered local inflammation was reduced, providing a competitive advantage to Escherichia species, possibly triggering a positive feedback loop that contributed to the taxonomic changes. ${ }^{167}$ Akkermansia was also found at similar abundances in non-diabetic control and metformintreated diabetic patients. ${ }^{167}$ Furthermore, functional analysis demonstrated significantly enhanced butyrate and propionate production potential in metformin-treated patients. This is of significance due to the potential of these SCFA to trigger intestinal gluconeogenesis. ${ }^{168} \quad 169$ Thus, characterisation of a metformin-associated human gut microbiome highlighted novel microbial mechanisms that contribute to the beneficial effects of the drug on host metabolism and provided evidence for the importance of controlling for metformin treatment once reporting functional and compositional shifts in the human microbiome.

What's more, microbial manipulation to treat insulin resistance has previously been described, whereby microbial faecal transplant from lean donors to male insulin-resistant subjects significantly improved peripheral insulin resistance associated with an altered small intestinal microbial composition. ${ }^{170}$

\section{PROBIOTICS AS DIABETIC THERAPIES}

It is now well established that there is enormous potential for human metabolic health in the manipulation of the gut microbiome. ${ }^{171}$ We have already discussed the inverse relationship between gut microbiota diversity and diabetic state; however, it is likely that certain keystone microbial taxa, alongside diversity, may be at the centre of this association. Aside from diet, probiotic administration currently represents the most effective and safest means of selectively altering the gut microbiome, ultimately with the intention to improve host health. ${ }^{78} 172$

\section{Probiotics for T1D}

It is well established that the microbiome can have a central role in the progression of T1D. ${ }^{173}$ However, it also appears to be true that we can alter the microbiome to delay onset or manage T1D pathogenesis. Three main targets have emerged for probiotic therapy in T1D; reduction or redirection of autoimmunity, increased $\beta$-cell proliferation and decreased $\beta$-cell apoptosis (figure 2).

The redirection of T-cell differentiation towards $\mathrm{T}_{\text {reg }}$ cells by probiotics, commensals and their metabolites has received some attention. A probiotic cocktail of three Lactobacillus plantarum strains was observed to significantly reduce inflammatory cytokines interferon (IFN) $\gamma$ and tumour necrosis factor- $\alpha$ production, while increasing anti-inflammatory interleukin-10 production in a mouse model of autoimmunity. ${ }^{174}$ The zwitterionic polysaccharide of $B$. fragilis (PSA) has also been implicated in a similar mechanism of anti-inflammatory protection for autoimmune disease-induced mice. ${ }^{175}{ }^{176}$ One study investigating the ability of two Lactobacillus spp. isolated from the GI tract of T1D-resistant mice found that administration of a Lactobacillus johnsonii delayed or prevented the onset of the disease in a BioBreeding model. ${ }^{177}$ The strain elicited this

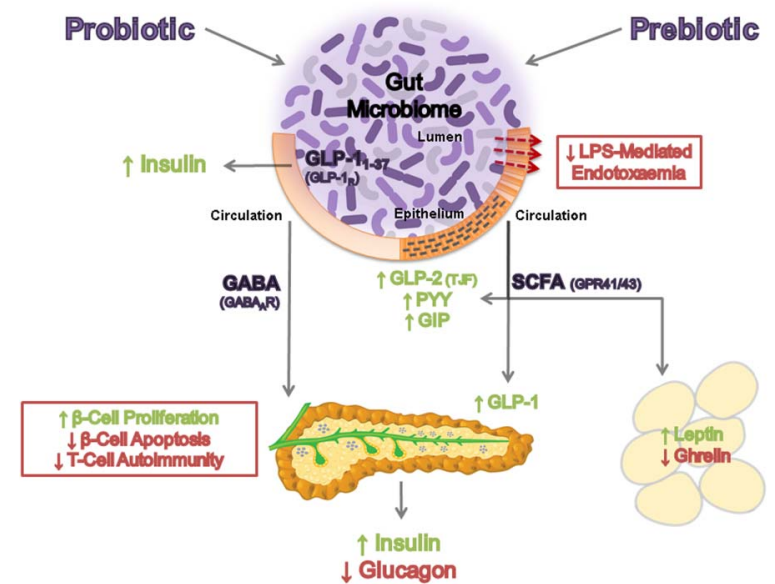

Figure 2 Putative mechanisms of action through which prebiotics and probiotic bacteria can impact on host metabolic health in type 1 and type 2 diabetes. Green and red texts indicate hormones, systems and actions that are upregulated and downregulated, respectively. LPS, lipopolysaccharide.

response by reducing host IFN $\gamma$ and iNOS levels, while also increasing claudin expression.

Conversely, some members of the gut microbiota have been shown to accelerate T1D. ${ }^{178}$ Segmented filamentous bacteria (SFB) are a Gram-positive, strict anaerobe taxonomic lineage within the Clostridiaceae family, which occupy the GI tract. ${ }^{179}$ These SFB are found tightly associated with the intestinal wall and, as a result, interact with the host immune system causing considerable differentiation towards inflammatory $\mathrm{T}_{\mathrm{H}} 17$ cells in the small intestinal lamina propria. ${ }^{180}$ While this type of immunostimulation can be extremely important to the development of a healthy host immune system, ${ }^{181}$ this same action can exacerbate autoimmunity in others that are genetically susceptible to T1D. ${ }^{182}$ Just one probiotic strain has previously been described as displaying anti-SFB activity in an immunocompromised mouse model. ${ }^{183}$ However, the mechanistic features proposed by the authors are those common to many probiotics - direct pathogen exclusion, antimicrobial activity or indirect activity through host immunostimulation ${ }^{184}{ }^{185}$-suggesting that SFB may be a potential target for probiotic therapy in T1D.

GABA is a metabolite produced from glutamate by the glutamate decarboxylase (GAD) pathway of many lactic acid bacteria, ${ }^{186}$ as a cellular response to acidic stress. ${ }^{187}$ In the host, GABA is produced by $\beta$-cells and acts as the main inhibitory neurotransmitter in the enteric and parasympathetic nervous systems, where it is involved in pain perception and stress. ${ }^{188}$ However, GABA has also been proposed as a potential therapy in T1D pathogenesis. ${ }^{189} 190$ GABA acts on $\beta$-cell receptors, $G_{A B A} R$ and $G_{A B A} R$, to increase insulin production and $\beta$-cell proliferation, while also reducing $\beta$-cell apoptosis. ${ }^{191}$ In addition, $\mathrm{CD}^{+}{ }^{+} \mathrm{T}$ cells have also been shown to harbour $\mathrm{GABA}_{\mathrm{A}} \mathrm{R}$, allowing the amino acid to interact with the immune system to effectively inhibit the inflammatory process involved in the progression of T1D. ${ }^{192} 193$ While these studies have investigated the use of injected or parenteral delivery of pure GABA, none have applied GABA-producing probiotics to a T1D model. The research to date investigating the bioactivity of GABA-producing probiotics has been solely focused on the gutbrain axis. ${ }^{194}$ However, selected probiotics can produce physiologically relevant levels GABA in faecal fermentations ${ }^{186}$ and, as such, should be considered potential T1D therapies warranting 
further investigation. One such study recently conducted within our group investigated the potential of an efficient GABA-producing Lactobacillus brevis in modifying host metabolism (Marques et al, in press). Following 5 weeks of probiotic intervention, healthy rat serum insulin was increased by $70 \%$ when compared with controls, while all other metabolic parameters were not significantly altered.

An intriguing novel avenue that is currently being explored in probiotic research is the engineering of commensal bacteria that can deliver bioactive molecules capable of managing or correcting a disease state. ${ }^{195}$ Due to their safety and the association that probiotics have with host intestinal mucosa, this system has been of considerable interest for vaccine delivery (for review, see Wells and Mercenier $\left.{ }^{186}{ }^{196}\right)$. Such treatments have been coined as synthetic signalling therapeutics. ${ }^{197}$ In a recent study, a recombinant probiotic Lactobacillus gasseri ATCC 33323 capable of secreting the inactive full length form of glucagonlike peptide-1 (GLP-1)(1-37) was designed. ${ }^{198}$ GLP-1 is a conserved mammalian peptide produced by neuroendocrine L-cells, which can lower postprandial glucose levels in T2D by stimulating insulin production and attenuating glucagon release. ${ }^{199}$ GLP-1 also acts systemically on the pancreas, liver, heart, stomach and brain, impacting on gastric emptying, satiety and lipid absorption and metabolism. ${ }^{200} 201$ As such, a plethora of drugs have been developed as GLP-1R agonists or blockers (gliptins) of DPP-4, the enzyme that cleaves active GLP-1 in circulation. ${ }^{202}$ Although GLP-1-based therapies have been entirely targeted towards the treatment of T2D, it has become clear that this full-length form of the peptide may have significant potential as a T1D therapy. GLP-1 was originally identified in its extended 1-37 form through cDNA cloning, ${ }^{203}$ although it was thought to have little bioactivity compared with the truncated GLP-1 (7-37 and 7-36), later discovered to be naturally produced by human $\beta$-cells. ${ }^{204}$ However, a recombinant GLP-1(137) producing $E$. coli has previously been shown to stimulate the production of $1 \mathrm{ng}$ insulin per millilitre of media in Caco-2 cells. ${ }^{205}$ Furthermore, the peptide has also been implicated in the conversion of crypt-located small intestinal epithelial cells to insulin $^{+} \beta$-like-cells ex vivo. ${ }^{206}$ Following STZ-induction of T1D in mice, Duan et al demonstrated that twice daily administration of a high dose $\left(1.6 \times 10^{10} \mathrm{CFU} / \mathrm{kg}\right)$ of this recombinant GLP-1(1-37) producing probiotic for 90 days resulted in $~ 30 \%$ restoration of insulin-production capabilities and significantly improved glucose metabolism. It was confirmed by immunofluorescent microscopy that this activity was through differentiation of intestinal crypt epithelial cells to $\beta$-like cells. Thus, GI tract-derived probiotics may serve as drug delivery or signalling systems, which could have downstream implications for diabetes. ${ }^{207} 208$

\section{Probiotics for T2D}

Much of the initial research around probiotics as T2D therapies has been through non-targeted approaches, assessing the ability of different lactobacilli and bifidobacteria to attenuate the effects of the disease state without any genuine molecular hypothesis prior to experimentation. ${ }^{209} 210$ More recent studies have progressed to implicating reduced inflammation, as a direct result of improved tight junction function preventing metabolic endotoxemia, as the source of the probiotic effect in diabetes therapy (figure 2). ${ }^{162} 211212$ This attenuation of low-grade inflammation undoubtedly plays a central role in T2D therapy; however, the manner in which this is achieved by probiotic bacteria is complex. The most compelling theories of the T2Dmicrobiome interaction have come from the Delzenne and Cani group of the University of Louvain (for review, see Cani et al). ${ }^{162} 204206-214$ The systematic research of this group has uncovered several metabolic targets for probiotic therapies, some of which we have previously discussed already.

The first and most thoroughly characterised targets for probiotic-mediated T2D therapy are the SCFA. SCFA-primarily acetate, butyrate and propionate-are thought to interact with the host enteroendocrine system by binding to GPR 41 and GPR43. ${ }^{214} 215$ These GPCRs in turn upregulate the expression of gut hormone precursor peptide proglucagon, ${ }^{216}$ GLP-1, ${ }^{217}$ GLP-2, ${ }^{95}$ gastric inhibitory polypeptide (GIP) and peptide YY (PYY) levels, ${ }^{218}$ as well as adipocyte-derived leptin, ${ }^{219}$ while downregulating ghrelin. ${ }^{220}$ As mentioned previously, GLP-1-based therapies have demonstrated great efficacy in treating patients presenting T2D, as well as dyslipidemia. However, microbiota-mediated modulation of these other gut hormones also has significant implications for metabolic health, affecting gut permeability, satiety, gastric emptying and food intake. ${ }^{221}$ Most recently, SCFAs have also been shown to act upon a cAMP-dependent mechanism, increasing intestinal gluconeogenesis, ${ }^{168}$ which is thought to contribute to satiety. ${ }^{222}$ While some studies have reported contrasting effects, this is likely a result of the varying actions of specific SCFA. As we have discussed previously, it appears that butyrate may be of most significance in the correction of metabolic endotoxemia disease states through proliferation of colonic epithelial cells and tight junction function. ${ }^{151}$ The importance of butyrate-producing bacteria in T2D development has been demonstrated by a recent human faecal transplant study. ${ }^{170}$ This research implicates enteric butyrateproducing bacteria, such as $R$. intestinalis, Faecalibacterium spp. and Eubacterium hallii, as potential probiotics for the alleviation of metabolic endotoxemia-induced T2D. ${ }^{223}$ There is also evidence that more classical probiotics, such as the commercial probiotic cocktail VSL\#3, can offer complete correction of weight gain and glucose intolerance associated with diet-induced T2D in mice. ${ }^{224}$ Moreover, a recent transcriptomics study in elderly patients demonstrated the ability of Lactobacillus rhamnosus GG to promote the relative expression of butyrateproducing Roseburia and Eubacterium genes within the metagenome. ${ }^{225}$ This could offer a more intriguing mechanism for the anti-inflammatory effects attributed to traditional probiotics in T2D therapy.

The second proposed target for T2D probiotic therapy is the eCB system, already discussed in relation to obesity. ${ }^{226} \mathrm{CB}_{1}$ is involved in gut permeability and modulation of the gut microbiota appears to yield similar responses to $\mathrm{CB}_{1}$ antagonists, upregulating expression and correcting the localisation of intestinal epithelial tight junction proteins, occludin and zonula occludens $1 .{ }^{227}$ Furthermore, supplementation with $2 \times 10^{8} \mathrm{CFU} /$ day of live A. muciniphila increased ileum 2-AG and 2palmitoylglycerol levels in mice fed a HF diet, which had the knock-on effect of reducing serum LPS content. ${ }^{73}$ The manner in which A. muciniphila alters eCB levels remains unclear, but the correlation is apparent and this may offer a mechanism of action for the beneficial metabolic effects attributed to the strain.

Finally, eCB-like lipids, such as $\mathrm{N}$-oleoylethanolamine, 2-oleoylglycerol (2-OG) and linoleoylethanolamine have been shown to interact with GPR119, a receptor that stimulates the host enteroendocrine system. ${ }^{228}$ Live A. muciniphila administration has demonstrated potential in altering 2-OG in mice fed a HF diet, contributing to tight junction function and significantly improving glucose metabolism, through stimulation of intestinal L-cell GLP-2 and GLP-1 production, respectively. ${ }^{73}$ In addition, 
the aforementioned increase in 2-AG production has been shown to potentiate anti-inflammatory effects from 2-OG, which could have further implications for correction of T2D. ${ }^{229}$

Additionally, GABA has also demonstrated potential in the treatment of T2D ex vivo ${ }^{230}$ and in vivo ${ }^{231}$ through the same pathways targeted for T1D. The former study, in which human pancreatic islets were excised, demonstrated that the GABA signalling pathway was hindered in T2D compared with healthy controls. ${ }^{230}$ However, GABA $\mathrm{R}$ agonist CPG55845 proved capable of stimulating insulin release from islets of both T2D and healthy control islets. Interestingly, Tian $e t a^{231}$ found that GABA therapy in a diet-induced obese model of T2D improved fasting glucose, glucose tolerance and insulin sensitivity through action on peripheral $\mathrm{GABA}_{\mathrm{A}} \mathrm{R}$-expressing immune cells. The study found that this in turn promoted a $\mathrm{CD} 4{ }^{+} \mathrm{Fox} 3^{+} \mathrm{T}_{\text {reg }}$ differentiation tone and, as a result, reduced T2D-associated inflammation. The potential for GABA-producing probiotics as T2D therapies certainly warrants preclinical investigation.

\section{LIMITATIONS AND RISKS OF MICROBIOME-MEDIATED THERAPIES}

While this review has highlighted the major advances and promising outcomes of current microbiome-metabolism research, it is important to comment on several limitations and potential risks associated with the science. In terms of limitations, as is the case for all personalised medicines, what works for me may not work for you. That is to say that an individual's genetics, specific diet, drug intake and endogenous microbiota may greatly impact on the efficacy of certain microbiome-mediated therapies. Moreover, some interventions may not only be ineffective in combating an individual's disease state, they may even be an additional risk factor. Certain avant-garde bacterial therapy procedures have demonstrated great potential in combating grave diseases: for example, the use of faecal microbiota transplants (FMTs) in the treatment of Clostridium difficile infection. However, with such efficacy comes risk. There have been several anecdotal reports and a single case study reporting increased weight gain following FMT from overweight donors ${ }^{232}$ mirroring preclinical outcomes. In addition, there has even been speculation that transplantation from a mentally unfit donor could induce the same phenotype in the recipient. Although it is unlikely that single or even multistrain bacterial therapies, which have undergone strenuous preclinical and clinical investigation, would transfer such deleterious attributes. However, it certainly warrants attention, and microbiomemediated therapies such as FMT will require significant regulation in order to reduce safety risks and ensure whole system host health.

\section{CONCLUSION}

The obesity epidemic has become a thorn in the side of a 21st-century global health crisis. Modern sequencing technologies have demonstrated that we are more microbe than man, in fact we always were, but the vast data now available to prove this are unarguable. The relationship between microbe and man is symbiotic, but all good relationships need work. And so we must nurture and feed ourselves healthy by indirectly feeding our microbes healthy too. The compelling evidence described in this review suggests that diet and environment can shape gut microbial composition and, in turn, gut microbial composition can have direct effects on human health. The ultimate goal is to permanently switch off this microbiota-influenced 'trip switch' determining metabolic endotoxemia in the host. This may be ambitious right now, but it is clear that certain strains of bacteria (and the pharmabiotics they produce) can have very positive effects on host metabolic health. Although these effects may seem a small solution to this epidemic, the future development of probiotics as medicines to treat metabolic disease or indeed prevent their onset is a promising proposal. The data described in this review bring us ever closer to discovering an 'antiobese microbiota', one that could prevent this obesity epidemic from polluting future global health.

\section{Main messages}

- While the gut microbiota is unique and essential for development, its composition can be influenced by many factors including health status and environment.

- 'Pharmabiotics' are bioactive microbial metabolites that confer a health benefit to the host.

- The gut microbiota are a modifiable target with the potential to progress or prevent the development of the metabolic syndrome.

\section{Current research questions}

- Can we eat ourselves healthy by feeding specific gut microbes?

- Is it possible to transplant a healthful 'lean-microbiota' to an obese host with the effect of alleviating symptoms of the metabolic syndrome?

- Can specific strains of bacteria be used as future therapies against diabetes or could potential disease-provoking strains be knocked out of the gut microbial ecosystem?

\section{Key references}

- Murphy EF, Cotter PD, Healy S, et al. Composition and energy harvesting capacity of the gut microbiota: relationship to diet, obesity and time in mouse models. Gut 2010;59:1635-42.

- Ridaura VK, Faith JJ, Rey FE, et al. Gut microbiota from twins discordant for obesity modulate metabolism in mice. Science 2013;341:1241214.

- Cani PD, Geurts L, Matamoros S, et al. Glucose metabolism: focus on gut microbiota, the endocannabinoid system and beyond. Diabetes Metab 2014;40:246-57.

- Everard A, Belzer C, Geurts L, et al. Cross-talk between Akkermansia muciniphila and intestinal epithelium controls diet-induced obesity. Proc Natl Acad Sci USA 2013;110:9066-71.

- Tian J, Dang HN, Yong J, et al. Oral treatment with gamma-aminobutyric acid improves glucose tolerance and insulin sensitivity by inhibiting inflammation in high fat diet-fed mice. PLoS ONE 2011;6:e25338. 


\section{Self assessment questions}

Please answer true or false to the below.

1. Metabolic endotoxemia is characterised by excess lipopolysaccharide in the blood.

2. The endocannabinoid system is comprised of an array of signalling peptides.

3. Akkermansia can improve host insulin resistance in mice.

4. The pharmabiotic $\gamma$-aminobutyric acid functions as an excitatory neurotransmitter in the central nervous system.

5. Prebiotics are live microorganisms that may offer a therapeutic means of managing metabolic health.

Contributors EP, PMR and CS wrote the manuscript. JFC, GFF, RPR and TGD made substantial contributions to the overall content of the manuscript, and all authors had responsibility for the final content.

Funding PMR is in receipt of a Teagasc Walsh Fellowship. This work is supported by the APC Microbiome Institute. The APC Microbiome Institute is a research centre funded by Science Foundation Ireland (SFI). This publication has emanated from research supported by a research grant from Science Foundation Ireland (SFI) under grant number SFI/12/RC/2273.

Competing interests None declared.

Provenance and peer review Commissioned; externally peer reviewed.

\section{REFERENCES}

1 Barker DJ, Osmond C. Infant mortality, childhood nutrition, and ischaemic heart disease in England and Wales. Lancet 1986;1:1077-81.

2 Barker DJP. The origins of the developmental origins theory. J Intern Med 2007:261:412-17.

3 Aagaard K, Ma J, Antony KM, et al. The placenta harbors a unique microbiome. Sci Transl Med 2014:6:237ra65.

4 Dominguez-Bello MG, Costello EK, Contreras M, et al. Delivery mode shapes the acquisition and structure of the initial microbiota across multiple body habitats in newborns. Proc Natl Acad Sci USA 2010;107:11971-5.

5 Biasucci G, Rubini M, Riboni $\mathrm{S}$, et al. Mode of delivery affects the bacterial community in the newborn gut. Early Hum Dev 2010;86(Suppl 1):13-15

6 Jakobsson HE, Abrahamsson TR, Jenmalm MC, et al. Decreased gut microbiota diversity, delayed Bacteroidetes colonisation and reduced Th1 responses in infants delivered by caesarean section. Gut 2014;63:559-66.

7 Thum C, Cookson AL, Otter DE, et al. Can nutritional modulation of materna intestinal microbiota influence the development of the infant gastrointestinal tract? J Nutr 2012;142:1921-8.

8 Schwartz S, Friedberg I, Ivanov IV, et al. A metagenomic study of diet-dependent interaction between gut microbiota and host in infants reveals differences in immune response. Genome Biol 2012;13:r32.

9 Fan W, Huo G, Li X, et al. Impact of diet in shaping gut microbiota revealed by a comparative study in infants during the six months of life. J Microbiol Biotechnol 2014;24:133-43.

10 Roger LC, Costabile A, Holland DT, et al. Examination of faecal Bifidobacterium populations in breast- and formula-fed infants during the first 18 months of life. Microbiology (Reading, Engl) 2010;156(Pt 11):3329-41.

11 Palmer C, Bik EM, DiGiulio DB, et al. Development of the human infant intestinal microbiota. PLoS Biol 2007:5:e177.

12 Borre YE, O'Keeffe GW, Clarke G, et al. Microbiota and neurodevelopmental windows: implications for brain disorders. Trends Mol Med 2014;20:509-18.

13 Eckburg PB, Bik EM, Bernstein CN, et al. Diversity of the human intestinal microbial flora. Science 2005;308:1635-8.

14 Guinane CM, Cotter PD. Role of the gut microbiota in health and chronic gastrointestinal disease: understanding a hidden metabolic organ. Therap Adv Gastroenterol 2013:6:295-308.

15 Turnbaugh PJ, Bäckhed F, Fulton L, et al. Diet-induced obesity is linked to marked but reversible alterations in the mouse distal gut microbiome. Cell Host Microbe 2008;3:213-23.

16 Bäckhed F, Manchester JK, Semenkovich CF, et al. Mechanisms underlying the resistance to diet-induced obesity in germ-free mice. Proc Natl Acad Sci USA 2007:104:979-84

17 Desbonnet L, Clarke G, Shanahan F, et al. Microbiota is essential for social development in the mouse. Mol Psychiatry 2014;19:146-8.
18 Lee YK, Mazmanian SK. Has the microbiota played a critical role in the evolution of the adaptive immune system? Science 2010;330:1768-73.

19 Macpherson AJ, Harris NL. Interactions between commensal intestinal bacteria and the immune system. Nat Rev Immunol 2004:4:478-85.

20 Falk PG, Hooper LV, Midtvedt T, et al. Creating and maintaining the gastrointestinal ecosystem: what we know and need to know from gnotobiology. Microbiol Mol Biol Rev 1998;62:1157-70.

21 Pollard M, Sharon N. Responses of the Peyer's patches in germ-free mice to antigenic stimulation. Infect Immun 1970;2:96-100.

22 Hoshi $\mathrm{H}$, Aijima $\mathrm{H}$, Horie $\mathrm{K}$, et al. Lymph follicles and germinal centers in popliteal lymph nodes and other lymphoid tissues of germ-free and conventional rats. Tohoku J Exp Med 1992;166:297-307.

23 Shanahan F. Therapeutic implications of manipulating and mining the microbiota. $J$ Physiol (Lond) 2009;587(Pt 17):4175-9.

24 Patterson E, Cryan JF, Fitzgerald GF, et al. Gut microbiota, the pharmabiotics they produce and host health. Proc Nutr Soc 2014;73:477-89.

25 Ryan PM, Ross RP, Fitzgerald GF, et al. Sugar-coated: exopolysaccharide producing lactic acid bacteria for food and human health applications. Food Funct 2015:6:679-93

26 Willing BP, Dicksved J, Halfvarson J, et al. A pyrosequencing study in twins shows that gastrointestinal microbial profiles vary with inflammatory bowel disease phenotypes. Gastroenterology 2010;139:1844-54.e1.

27 Tong $M, L i X$, Wegener Parfrey $L$, et al. A modular organization of the human intestinal mucosal microbiota and its association with inflammatory bowel disease. PLOS ONE 2013;8:e80702.

28 Manichanh C, Borruel N, Casellas F, et al. The gut microbiota in IBD. Nat Rev Gastroenterol Hepatol 2012;9:599-608.

29 Manichanh C, Rigottier-Gois L, Bonnaud E, et al. Reduced diversity of faeca microbiota in Crohn's disease revealed by a metagenomic approach. Gut 2006;55:205-11.

30 Turnbaugh PJ, Hamady M, Yatsunenko T, et al. A core gut microbiome in obese and lean twins. Nature 2009:457:480-4.

31 Ley RE, Turnbaugh PJ, Klein S, et al. Microbial ecology: human gut microbes associated with obesity. Nature 2006;444:1022-3.

32 Schwiertz A, Taras D, Schäfer K, et al. Microbiota and SCFA in lean and overweight healthy subjects. Obesity (Silver Spring) 2010;18:190-5.

33 Zhang H, DiBaise JK, Zuccolo A, et al. Human gut microbiota in obesity and after gastric bypass. Proc Natl Acad Sci USA 2009;106:2365-70.

34 de Goffau MC, Luopajärvi K, Knip M, et al. Fecal microbiota composition differs between children with beta-cell autoimmunity and those without. Diabetes 2013:62:1238-44.

35 Brown CT, Davis-Richardson AG, Giongo A, et al. Gut microbiome metagenomics analysis suggests a functional model for the development of autoimmunity for type 1 diabetes. PLOS ONE 2011;6:e25792.

36 Murri M, Leiva I, Gomez-Zumaquero JM, et al. Gut microbiota in children with type 1 diabetes differs from that in healthy children: a case-control study. BMC Med 2013;11:46.

37 Qin J, Li Y, Cai Z, et al. A metagenome-wide association study of gut microbiota in type 2 diabetes. Nature 2012;490:55-60.

38 Zhang $X$, Shen $D$, Fang $Z$, et al. Human gut microbiota changes reveal the progression of glucose intolerance. PLOS ONE 2013;8:e71108.

39 Hu B, Elinav E, Huber $S$, et al. Microbiota-induced activation of epithelial IL-6 signaling links inflammasome-driven inflammation with transmissible cancer. Proc Natl Acad Sci USA 2013;110:9862-7.

40 Elinav E, Nowarski R, Thaiss CA, et al. Inflammation-induced cancer: crosstalk between tumours, immune cells and microorganisms. Nat Rev Cancer 2013;13:759-71.

41 Garrett WS. Cancer and the microbiota. Science 2015;348:80-6

42 Irrazábal T, Belcheva A, Girardin SE, et al. The multifaceted role of the intestinal microbiota in colon cancer. Mol Cell 2014;54:309-20.

43 Dinan TG, Stanton C, Cryan JF. Psychobiotics: a novel class of psychotropic. Biol Psychiatry 2013;74:720-6.

44 Naseribafrouei A, Hestad K, Avershina E, et al. Correlation between the human fecal microbiota and depression. Neurogastroenterol Motil 2014;26:1155-62.

45 Hsiao EY, McBride SW, Hsien S, et al. Microbiota modulate behavioral and physiological abnormalities associated with neurodevelopmental disorders. Cell 2013;155:1451-63.

46 de Theije CGM, Wu JB, da Silva SL, et al. Pathways underlying the gut-to-brain connection in autism spectrum disorders as future targets for disease management. Eur J Pharmacol 2011;668(Suppl 1):S70-80.

47 Finegold SM, Dowd SE, Gontcharova V, et al. Pyrosequencing study of fecal microflora of autistic and control children. Anaerobe 2010;16:444-53.

48 Dinan TG, Stilling RM, Stanton C, et al. Collective unconscious: How gut microbes shape human behavior. J Psychiatr Res 2015;63:1-9.

49 O'Mahony SM, Marchesi JR, Scully P, et al. Early life stress alters behavior, immunity, and microbiota in rats: implications for irritable bowel syndrome and psychiatric illnesses. Biol Psychiatry 2009;65:263-7. 
50 Neufeld KM, Kang N, Bienenstock J, et al. Reduced anxiety-like behavior and central neurochemical change in germ-free mice. Neurogastroenterol Motil 2011;23:255-64, e119.

51 Scheperjans F, Aho V, Pereira PA, et al. Gut microbiota are related to Parkinson's disease and clinical phenotype. Mov Disord 2015;30:350-8.

52 Mujico JR, Baccan GC, Gheorghe A, et al. Changes in gut microbiota due to supplemented fatty acids in diet-induced obese mice. Br J Nutr 2013;110: 711-20.

53 Murphy EF, Cotter PD, Healy S, et al. Composition and energy harvesting capacity of the gut microbiota: relationship to diet, obesity and time in mouse models. Gut 2010;59:1635-42.

54 de Wit NJ, Derrien M, Bosch-Vermeulen $\mathrm{H}$, et al. Saturated fat stimulates obesity and hepatic steatosis and affects gut microbiota composition by an enhanced overflow of dietary fat to the distal intestine. Am J Physiol Gastrointest Liver Physiol 2012;303:G589-99.

55 Ravussin Y, Koren 0, Spor A, et al. Responses of gut microbiota to diet composition and weight loss in lean and obese mice. Obesity (Silver Spring) 2012;20:738-47.

56 Patterson E, O'Doherty RM, Murphy EF, et al. Impact of dietary fatty acids on metabolic activity and host intestinal microbiota composition in C57BL/6J mice. $\mathrm{Br}$ J Nutr 2014;111:1905-17.

57 Marques TM, Wall R, O'Sullivan 0, et al. Dietary trans-10, cis-12-conjugated linoleic acid alters fatty acid metabolism and microbiota composition in mice. $\mathrm{Br} J$ Nutr 2015;113:728-38.

58 Daniel H, Moghaddas Gholami A, Berry D, et al. High-fat diet alters gut microbiota physiology in mice. ISME J 2014;8:295-308.

59 De Filippo C, Cavalieri D, Di Paola M, et al. Impact of diet in shaping gut microbiota revealed by a comparative study in children from Europe and rural Africa. Proc Natl Acad Sci USA 2010;107:14691-6.

60 Turnbaugh PJ, Ridaura VK, Faith JJ, et al. The effect of diet on the human gut microbiome: a metagenomic analysis in humanized gnotobiotic mice. Sci Trans/ Med 2009:1:6ra14.

$61 \mathrm{Xu}$ ZJ, Knight R. Dietary effects on human gut microbiome diversity. Br J Nutr 2015;113:S1-5.

62 Graf D, Di Cagno R, Fåk F, et al. Contribution of diet to the composition of the human gut microbiota. Microb Ecol Health Dis 2015;26:26164.

63 Flint HJ, Scott KP, Louis P, et al. The role of the gut microbiota in nutrition and health. Nat Rev Gastroenterol Hepatol 2012;9:577-89.

64 Zhang $\mathrm{CH}$, Zhang $\mathrm{MH}$, Pang $\mathrm{XY}$, et al. Structural resilience of the gut microbiota in adult mice under high-fat dietary perturbations. ISME $J$ 2012;6:1848-57.

65 Kong LC, Holmes BA, Cotillard A, et al. Dietary patterns differently associate with inflammation and gut microbiota in overweight and obese subjects. PLOS ONE 2014;9:e109434.

66 Clarke SF, Murphy EF, O'Sullivan 0, et al. Exercise and associated dietary extremes impact on gut microbial diversity. Gut 2014;63:1913-20.

67 Le Chatelier E, Nielsen T, Qin J, et al. Richness of human gut microbiome correlates with metabolic markers. Nature 2013:500:541-6.

68 Oh DY, Talukdar S, Bae EJ, et al. GPR120 is an omega-3 fatty acid receptor mediating potent anti-inflammatory and insulin-sensitizing effects. Cell 2010;142:687-98.

69 Caesar R, Tremaroli V, Kovatcheva-Datchary P, et al. Crosstalk between gut microbiota and dietary lipids aggravates WAT inflammation through TLR signaling. Cell Metab 2015;22:658-68.

70 Devkota S, Wang Y, Musch MW, et al. Dietary-fat-induced taurocholic acid promotes pathobiont expansion and colitis in $1110-/-$ mice. Nature 2012:487:104-8

71 Cani PD, Amar J, Iglesias MA, et al. Metabolic endotoxemia initiates obesity and insulin resistance. Diabetes 2007:56:1761-72.

72 Henao-Mejia J, Elinav E, Jin C, et al. Inflammasome-mediated dysbiosis regulates progression of NAFLD and obesity. Nature 2012:482:179-85.

73 Everard A, Belzer C, Geurts L, et al. Cross-talk between Akkermansia muciniphila and intestinal epithelium controls diet-induced obesity. Proc Natl Acad Sci USA 2013;110:9066-71.

74 Karlsson CL, Onnerfält J, Xu J, et al. The microbiota of the gut in preschool children with normal and excessive body weight. Obesity (Silver Spring) 2012;20:2257-61.

75 Wu M, McNulty NP, Rodionov DA, et al. Genetic determinants of in vivo fitness and diet responsiveness in multiple human gut Bacteroides. Science 2015;350: aac5992.

76 Faith JJ, Guruge JL, Charbonneau M, et al. The long-term stability of the human gut microbiota. Science 2013;341:1237439.

77 David LA, Maurice CF, Carmody RN, et al. Diet rapidly and reproducibly alters the human gut microbiome. Nature 2014;505:559-63.

78 Carmody RN, Gerber GK, Luevano JM, Jr., et al. Diet dominates host genotype in shaping the murine gut microbiota. Cell Host Microbe 2015;17:72-84.

79 Yatsunenko T, Rey FE, Manary MJ, et al. Human gut microbiome viewed across age and geography. Nature 2012;486:222-7.
80 Ussar S, Griffin NW, Bezy 0, et al. Interactions between gut microbiota, host genetics and diet modulate the predisposition to obesity and metabolic syndrome. Cell Metab 2015;22:516-30.

81 Bolnick DI, Snowberg LK, Hirsch PE, et al. Individual diet has sex-dependent effects on vertebrate gut microbiota. Nat Commun 2014;5:4500.

82 Organization WH. Fact sheet: obesity and overweight. 2006. http://www whoint/ dietphysicalactivity/publications/facts/obesity/en/ (accessed 3 Jan 2005).

83 Kelly T, Yang W, Chen CS, et al. Global burden of obesity in 2005 and projections to 2030. Int J Obes 2008;32:1431-7.

84 Turnbaugh PJ, Ley RE, Mahowald MA, et al. An obesity-associated gut microbiome with increased capacity for energy harvest. Nature 2006;444:1027-31.

$85 \mathrm{Xu} \mathrm{J,} \mathrm{Bjursell} \mathrm{MK,} \mathrm{Himrod} \mathrm{J,} \mathrm{et} \mathrm{al.} \mathrm{A} \mathrm{genomic} \mathrm{view} \mathrm{of} \mathrm{the} \mathrm{human-Bacteroides}$ thetaiotaomicron symbiosis. Science 2003;299:2074-6.

86 Sonnenburg JL, Xu J, Leip DD, et al. Glycan foraging in vivo by an intestine-adapted bacterial symbiont. Science 2005;307:1955-9.

$87 \mathrm{Hu}$ GX, Chen GR, Xu H, et al. Activation of the AMP activated protein kinase by short-chain fatty acids is the main mechanism underlying the beneficial effect of a high fiber diet on the metabolic syndrome. Med Hypotheses 2010;74:123-6.

88 Gao Z, Yin J, Zhang J, et al. Butyrate improves insulin sensitivity and increases energy expenditure in mice. Diabetes 2009;58:1509-17.

89 Blouin JM, Penot G, Collinet $\mathrm{M}$, et al. Butyrate elicits a metabolic switch in human colon cancer cells by targeting the pyruvate dehydrogenase complex. Int I Cancer 2011;128:2591-601.

90 Scharlau D, Borowicki A, Habermann N, et al. Mechanisms of primary cancer prevention by butyrate and other products formed during gut flora-mediated fermentation of dietary fibre. Mutat Res 2009;682:39-53.

91 Tang $Y$, Chen $Y$, Jiang $H$, et al. G-protein-coupled receptor for short-chain fatty acids suppresses colon cancer. Int J Cancer 2011;128:847-56.

92 Bäckhed $\mathrm{F}$, Ding $\mathrm{H}$, Wang $\mathrm{T}$, et al. The gut microbiota as an environmental factor that regulates fat storage. Proc Natl Acad Sci USA 2004;101:15718-23.

93 Cani PD, Neyrinck AM, Fava F, et al. Selective increases of bifidobacteria in gut microflora improve high-fat-diet-induced diabetes in mice through a mechanism associated with endotoxaemia. Diabetologia 2007;50:2374-83.

94 Cani PD, Bibiloni $R$, Knauf $C$, et al. Changes in gut microbiota control metabolic endotoxemia-induced inflammation in high-fat diet-induced obesity and diabetes in mice. Diabetes 2008;57:1470-81.

95 Cani PD, Possemiers S, Van de Wiele T, et al. Changes in gut microbiota control inflammation in obese mice through a mechanism involving GLP-2-driven improvement of gut permeability. Gut 2009;58:1091-103.

96 Neyrinck AM, Van Hée VF, Piront $N$, et al. Wheat-derived arabinoxylan oligosaccharides with prebiotic effect increase satietogenic gut peptides and reduce metabolic endotoxemia in diet-induced obese mice. Nutr Diabetes 2012;2: e28.

97 Brun P, Castagliuolo I, Di Leo V, et al. Increased intestinal permeability in obese mice: new evidence in the pathogenesis of nonalcoholic steatohepatitis. Am J Physiol Gastrointest Liver Physiol 2007;292:G518-25.

98 de La Serre CB, Ellis CL, Lee J, et al. Propensity to high-fat diet-induced obesity in rats is associated with changes in the gut microbiota and gut inflammation. Am J Physiol Gastrointest Liver Physiol 2010;299:G440-8.

99 Everard $A$, Lazarevic V, Derrien $M$, et al. Responses of gut microbiota and glucose and lipid metabolism to prebiotics in genetic obese and diet-induced leptin-resistant mice. Diabetes 2011:60:2775-86.

100 Turner JR. Intestinal mucosal barrier function in health and disease. Nat Rev Immunol 2009;9:799-809.

101 Ridaura VK, Faith JJ, Rey FE, et al. Gut microbiota from twins discordant for obesity modulate metabolism in mice. Science 2013;341:1241214

102 Derrien M, Collado MC, Ben-Amor K, et al. The Mucin degrader Akkermansia muciniphila is an abundant resident of the human intestinal tract. App/ Environ Microbiol 2008;74:1646-8.

103 Lukovac S, Belzer C, Pellis L, et al. Differential modulation by Akkermansia muciniphila and Faecalibacterium prausnitzii of host peripheral lipid metabolism and histone acetylation in mouse gut organoids. MBio 2014;5:pii: e01438-14.

104 Derrien M, Vaughan EE, Plugge CM, et al. Akkermansia muciniphila gen. nov., sp. nov., a human intestinal mucin-degrading bacterium. Int I Syst Evol Microbiol 2004;54(Pt 5):1469-76.

105 Derrien M, Van Baarlen P, Hooiveld G, et al. Modulation of mucosal immune response, tolerance, and proliferation in mice colonized by the mucin-degrader Akkermansia muciniphila. Front Microbiol 2011;2:166.

106 Hansen $\mathrm{CH}$, Krych L, Nielsen DS, et al. Early life treatment with vancomycin propagates Akkermansia muciniphila and reduces diabetes incidence in the NOD mouse. Diabetologia 2012;55:2285-94.

107 Dubourg G, Lagier JC, Armougom F, et al. High-level colonisation of the human gut by Verrucomicrobia following broad-spectrum antibiotic treatment. Int $\mathrm{J}$ Antimicrob Agents 2013;41:149-55.

108 lapichino G, Callegari ML, Marzorati S, et al. Impact of antibiotics on the gut microbiota of critically ill patients. J Med Microbiol 2008;57:1007-14. 
109 Caputo A, Dubourg G, Croce 0, et al. Whole-genome assembly of Akkermansia muciniphila sequenced directly from human stool. Biol Direct 2015;10;5.

110 Kang CS, Ban M, Choi EJ, et al. Extracellular vesicles derived from gut microbiota, especially Akkermansia muciniphila, protect the progression of dextran sulfate sodium-induced colitis. PLOS ONE 2013;8:e76520.

111 Ganesh BP, Klopfleisch R, Loh G, et al. Commensal Akkermansia muciniphila exacerbates gut inflammation in Salmonella Typhimurium-infected gnotobiotic mice. PLOS ONE 2013:8:e74963.

112 Sokol $\mathrm{H}$, Pigneur $B$, Watterlot $L$, et al. Faecalibacterium prausnitzii is an anti-inflammatory commensal bacterium identified by gut microbiota analysis of Crohn disease patients. Proc Natl Acad Sci USA 2008;105:16731-6.

113 Furet JP, Kong LC, Tap J, et al. Differential adaptation of human gut microbiota to bariatric surgery-induced weight loss: links with metabolic and low-grade inflammation markers. Diabetes 2010;59:3049-57.

114 Remely M, Aumueller E, Merold C, et al. Effects of short chain fatty acid producing bacteria on epigenetic regulation of FFAR3 in type 2 diabetes and obesity. Gene 2014;537:85-92.

115 Bonnema A, Kelly J, Ryan S, et al. SATIN (Satiety Innovation) project: dietary supplementation with type 3 resistant starch induces distinct changes in gut microbiota of overweight human volunteers. FASEB J 2015;29(1 Suppl):744.2.

116 Martín R, Chain F, Miquel $\mathrm{S}$, et al. The commensal bacterium Faecalibacterium prausnitzii is protective in DNBS-induced chronic moderate and severe colitis models. Inflamm Bowel Dis 2014;20:417-30.

117 Martín R, Miquel S, Chain F, et al. Faecalibacterium prausnitzii prevents physiological damages in a chronic low-grade inflammation murine model. BMC Microbiol 2015;15:67.

118 Di Marzo V, Goparaju SK, Wang L, et al. Leptin-regulated endocannabinoids are involved in maintaining food intake. Nature 2001;410:822-5.

119 Duncan M, Davison JS, Sharkey KA. Review article: endocannabinoids and their receptors in the enteric nervous system. Aliment Pharmacol Ther 2005;22:667-83.

120 Moran CP, Shanahan F. Gut microbiota and obesity: role in aetiology and potential therapeutic target. Best Pract Res Clin Gastroenterol 2014;28:585-97.

121 Mackie K. Cannabinoid receptors: where they are and what they do. J Neuroendocrinol 2008;20(Suppl 1):10-14.

122 Patel KD, Davison JS, Pittman QJ, et al. Cannabinoid CB(2) receptors in health and disease. Curr Med Chem 2010;17:1394-410.

123 Izzo AA, Piscitelli F, Capasso R, et al. Peripheral endocannabinoid dysregulation in obesity: relation to intestinal motility and energy processing induced by food deprivation and re-feeding. Br J Pharmacol 2009;158:451-61.

124 Trillou CR, Delgorge C, Menet C, et al. CB1 cannabinoid receptor knockout in mice leads to leanness, resistance to diet-induced obesity and enhanced leptin sensitivity. Int J Obes 2004;28:640-8.

125 Muccioli GG, Naslain D, Bäckhed F, et al. The endocannabinoid system links gut microbiota to adipogenesis. Mol Syst Biol 2010;6:392.

126 Atkinson MA, Eisenbarth GS, Michels AW. Type 1 diabetes. Lancet 2014;383:69-82.

127 Mallone R, Martinuzzi E, Blancou P, et al. CD8+ T-cell responses identify beta-cell autoimmunity in human type 1 diabetes. Diabetes 2007;56:613-21.

128 Barker JM, Triolo TM, Aly TA, et al. Two single nucleotide polymorphisms identify the highest-risk diabetes HLA genotype: potential for rapid screening. Diabetes 2008;57:3152-5.

129 Ziegler AG, Nepom GT. Prediction and pathogenesis in type 1 diabetes. Immunity 2010;32:468-78.

130 Todd JA, Walker NM, Cooper JD, et al. Robust associations of four new chromosome regions from genome-wide analyses of type 1 diabetes. Nat Genet 2007;39:857-64.

131 Barrett JC, Clayton DG, Concannon P, et al. Genome-wide association study and meta-analysis find that over 40 loci affect risk of type 1 diabetes. Nat Genet 2009; $41: 703-7$

132 Redondo MJ, Yu L, Hawa M, et al. Heterogeneity of type I diabetes: analysis of monozygotic twins in Great Britain and the United States. Diabetologia 2001;44:354-62.

133 Tuomilehto J. The emerging global epidemic of type 1 diabetes. Curr Diab Rep 2013:13:795-804

134 Gillespie KM, Bain SC, Barnett AH, et al. The rising incidence of childhood type 1 diabetes and reduced contribution of high-risk HLA haplotypes. Lancet 2004;364:1699-700.

135 Cardwell CR, Stene LC, Joner $G$, et al. Caesarean section is associated with an increased risk of childhood-onset type 1 diabetes mellitus: a meta-analysis of observational studies. Diabetologia 2008;51:726-35.

136 Wen L, Ley RE, Volchkov PY, et al. Innate immunity and intestinal microbiota in the development of Type 1 diabetes. Nature 2008:455:1109-13.

137 King C, Sarvetnick N. The incidence of type-1 diabetes in NOD mice is modulated by restricted flora not germ-free conditions. PLOS ONE 2011;6:e17049.

138 Brugman S, Klatter FA, Visser JT, et al. Antibiotic treatment partially protects against type 1 diabetes in the Bio-Breeding diabetes-prone rat. Is the gut flora involved in the development of type 1 diabetes? Diabetologia 2006;49: 2105-8.

139 Roesch LF, Lorca GL, Casella G, et al. Culture-independent identification of gut bacteria correlated with the onset of diabetes in a rat model. ISME J 2009;3:536-48.

140 Patterson E, Marques TM, O'Sullivan 0, et al. Streptozotocin-induced type-1-diabetes disease onset in Sprague-Dawley rats is associated with an altered intestinal microbiota composition and decreased diversity. Microbiology (Reading, Engl) 2015;161(Pt 1):182-93.

141 Neu J, Reverte CM, Mackey AD, et al. Changes in intestinal morphology and permeability in the biobreeding rat before the onset of type 1 diabetes. J Pediatr Gastroenterol Nutr 2005;40:589-95.

142 Bosi E, Molteni L, Radaelli MG, et al. Increased intestinal permeability precedes clinical onset of type 1 diabetes. Diabetologia 2006;49:2824-7.

143 Sapone A, de Magistris L, Pietzak M, et al. Zonulin upregulation is associated with increased gut permeability in subjects with type 1 diabetes and their relatives. Diabetes 2006;55:1443-9.

144 Graham S, Courtois P, Malaisse WJ, et al. Enteropathy precedes type 1 diabetes in the BB rat. Gut 2004:53:1437-44.

145 Vaarala O. Leaking gut in type 1 diabetes. Curr Opin Gastroenterol 2008;24:701-6.

146 Barcelo A, Claustre J, Moro F, et al. Mucin secretion is modulated by luminal factors in the isolated vascularly perfused rat colon. Gut 2000;46: 218-24.

147 Burger-van Paassen N, Vincent A, Puiman PJ, et al. The regulation of intestinal mucin MUC2 expression by short-chain fatty acids: implications for epithelial protection. Biochem J 2009;420:211-19.

148 Finnie IA, Dwarakanath $A D$, Taylor $B A$, et al. Colonic mucin synthesis is increased by sodium butyrate. Gut 1995;36:93-9.

149 Shimotoyodome A, Meguro S, Hase T, et al. Short chain fatty acids but not lactate or succinate stimulate mucus release in the rat colon. Comp Biochem Physiol $A$ Mol Integr Physiol 2000;125:525-31.

150 Peng L, He Z, Chen W, et al. Effects of butyrate on intestinal barrier function in a Caco-2 cell monolayer model of intestinal barrier. Pediatr Res 2007;61:37-41.

151 Peng L, Li ZR, Green RS, et al. Butyrate enhances the intestinal barrier by facilitating tight junction assembly via activation of AMP-activated protein kinase in Caco-2 cell monolayers. J Nutr 2009;139:1619-25.

152 Hamer HM, Jonkers $\mathrm{D}$, Venema $\mathrm{K}$, et al. Review article: the role of butyrate on colonic function. Aliment Pharmacol Ther 2008;27:104-19.

153 Louis $\mathrm{P}$, Flint HJ. Diversity, metabolism and microbial ecology of butyrate-producing bacteria from the human large intestine. FEMS Microbiol Lett 2009;294:1-8.

154 Pryde SE, Duncan SH, Hold GL, et al. The microbiology of butyrate formation in the human colon. FEMS Microbiol Lett 2002;217:133-9.

155 Giongo A, Gano KA, Crabb DB, et al. Toward defining the autoimmune microbiome for type 1 diabetes. ISME J 2011:5:82-91.

156 Endesfelder D, zu Castell W, Ardissone A, et al. Compromised gut microbiota networks in children with anti-islet cell autoimmunity. Diabetes 2014;63:2006-14.

157 Kostic AD, Gevers D, Siljander H, et al. The dynamics of the human infant gut microbiome in development and in progression toward type 1 diabetes. Cell Host Microbe 2015;17:260-73.

158 Johnson AM, Olefsky JM. The origins and drivers of insulin resistance. Cell 2013;152:673-84.

159 Pussinen PJ, Havulinna AS, Lehto $\mathrm{M}$, et al. Endotoxemia is associated with an increased risk of incident diabetes. Diabetes Care 2011;34:392-7.

160 Lassenius Ml, Pietiläinen $\mathrm{KH}$, Kaartinen $\mathrm{K}$, et al. Bacterial endotoxin activity in human serum is associated with dyslipidemia, insulin resistance, obesity, and chronic inflammation. Diabetes Care 2011;34:1809-15.

161 Serino $M$, Luche $E$, Gres $S$, et al. Metabolic adaptation to a high-fat diet is associated with a change in the gut microbiota. Gut 2012;61:543-53.

162 Amar J, Chabo C, Waget A, et al. Intestinal mucosal adherence and translocation of commensal bacteria at the early onset of type 2 diabetes: molecular mechanisms and probiotic treatment. EMBO Mol Med 2011;3:559-72.

163 Amar J, Serino M, Lange $C$, et al. Involvement of tissue bacteria in the onset of diabetes in humans: evidence for a concept. Diabetologia 2011;54:3055-61.

164 Tilg H, Moschen AR. Microbiota and diabetes: an evolving relationship. Gut 2014;63:1513-21.

165 Shin NR, Lee JC, Lee HY, et al. An increase in the Akkermansia spp. population induced by metformin treatment improves glucose homeostasis in diet-induced obese mice. Gut 2014;63:727-35.

166 Lee $\mathrm{H}$, Ko G. Effect of metformin on metabolic improvement and gut microbiota. Appl Environ Microbiol 2014;80:5935-43.

167 Forslund K, Hildebrand F, Nielsen T, et al. Disentangling type 2 diabetes and metformin treatment signatures in the human gut microbiota. Nature $2015 ; 528: 262-6$. 
168 De Vadder F, Kovatcheva-Datchary P, Goncalves D, et al. Microbiota-generated metabolites promote metabolic benefits via gut-brain neural circuits. Cell 2014;156:84-96.

169 Croset M, Rajas F, Zitoun C, et al. Rat small intestine is an insulin-sensitive gluconeogenic organ. Diabetes 2001;50:740-6.

170 Vrieze A, Van Nood E, Holleman F, et al. Transfer of intestinal microbiota from lean donors increases insulin sensitivity in individuals with metabolic syndrome. Gastroenterology 2012;143:913-6.e7.

171 Tremaroli V, Bäckhed F. Functional interactions between the gut microbiota and host metabolism. Nature 2012;489:242-9.

172 Hill C, Guarner F, Reid G, et al. Expert consensus document. The International Scientific Association for Probiotics and Prebiotics consensus statement on the scope and appropriate use of the term probiotic. Nat Rev Gastroenterol Hepatol 2014;11:506-14.

173 Dunne JL, Triplett EW, Gevers D, et al. The intestinal microbiome in type 1 diabetes. Clin Exp Immunol 2014;177:30-7.

174 Lavasani S, Dzhambazov B, Nouri M, et al. A novel probiotic mixture exerts a therapeutic effect on experimental autoimmune encephalomyelitis mediated by IL-10 producing regulatory T cells. PLOS ONE 2010;5:e9009.

175 Ochoa-Repáraz J, Mielcarz DW, Ditrio LE, et al. Central nervous system demyelinating disease protection by the human commensal Bacteroides fragilis depends on polysaccharide A expression. J Immunol 2010;185:4101-8.

176 Ochoa-Repáraz J, Mielcarz DW, Wang Y, et al. A polysaccharide from the human commensal Bacteroides fragilis protects against CNS demyelinating disease. Mucosal Immunol 2010;3:487-95.

177 Valladares R, Sankar D, Li N, et al. Lactobacillus johnsonii N6. 2 mitigates the development of type 1 diabetes in BB-DP rats. PLOS ONE 2010;5:e10507.

178 Hara N, Alkanani AK, Ir $D$, et al. The role of the intestinal microbiota in type 1 diabetes. Clin Immunol 2013;146:112-19.

179 Umesaki Y, Okada Y, Matsumoto S, et al. Segmented filamentous bacteria are indigenous intestinal bacteria that activate intraepithelial lymphocytes and induce MHC class II molecules and fucosyl asialo GM1 glycolipids on the small intestinal epithelial cells in the ex-germ-free mouse. Microbiol Immunol 1995;39:555-62.

180 Ivanov II, Atarashi K, Manel N, et al. Induction of intestinal Th17 cells by segmented filamentous bacteria. Cell 2009;139:485-98.

181 Mazmanian SK. Gut immune balance is as easy as S-F-B. Immunity 2009;31:536-8.

182 Wu HJ, Ivanov II, Darce J, et al. Gut-residing segmented filamentous bacteria drive autoimmune arthritis via T helper 17 cells. Immunity 2010;32:815-27.

183 Fuentes S, Egert M, Jimenez-Valera $\mathrm{M}$, et al. A strain of Lactobacillus plantarum affects segmented filamentous bacteria in the intestine of immunosuppressed mice. FEMS Microbiol Ecol 2008;63:65-72.

184 Bron PA, van Baarlen P, Kleerebezem M. Emerging molecular insights into the interaction between probiotics and the host intestinal mucosa. Nat Rev Microbiol 2012;10:66-78.

185 Lebeer S, Vanderleyden J, De Keersmaecker SCJ. Host interactions of probiotic bacterial surface molecules: comparison with commensals and pathogens. Nat Rev Microbiol 2010;8:171-84.

186 Barrett E, Ross RP, O'Toole PW, et al. gamma-Aminobutyric acid production by culturable bacteria from the human intestine. J App/ Microbiol 2012;113:411-17.

187 Feehily C, Karatzas KA. Role of glutamate metabolism in bacterial responses towards acid and other stresses. J App/ Microbiol 2013;114:11-24.

188 O'Leary OF, Felice D, Galimberti S, et al. GABAB(1) receptor subunit isoforms differentially regulate stress resilience. Proc Natl Acad Sci USA 2014;111:15232-7.

189 Soltani N, Qiu H, Aleksic M, et al. GABA exerts protective and regenerative effects on islet beta cells and reverses diabetes. Proc Natl Acad Sci USA 2011; 108:11692-7.

190 Tian J, Dang H, Kaufman DL. Combining antigen-based therapy with GABA treatment synergistically prolongs survival of transplanted B-cells in diabetic NOD mice. PLOS ONE 2011;6:e25337.

191 Tian J, Dang H, Chen Z, et al. gamma-Aminobutyric acid regulates both the survival and replication of human beta-cells. Diabetes 2013;62:3760-5.

192 Tian J, Lu Y, Zhang H, et al. $\gamma$-aminobutyric acid inhibits T cell autoimmunity and the development of inflammatory responses in a mouse type 1 diabetes model. $J$ Immunol 2004;173:5298-304.

193 Jin Z, Mendu SK, Birnir B. GABA is an effective immunomodulatory molecule. Amino Acids 2013:45:87-94.

194 Bravo JA, Forsythe P, Chew MV, et al. Ingestion of Lactobacillus strain regulates emotional behavior and central GABA receptor expression in a mouse via the vagus nerve. Proc Natl Acad Sci USA 2011;108:16050-5.

195 Goh YL, He H, March JC. Engineering commensal bacteria for prophylaxis against infection. Curr Opin Biotechnol 2012;23:924-30.

196 Wells JM, Mercenier A. Mucosal delivery of therapeutic and prophylactic molecules using lactic acid bacteria. Nat Rev Microbiol 2008;6:349-62.
197 Aurand TC, Russell MS, March JC. Synthetic signaling networks for therapeutic applications. Curr Opin Biotechnol 2012;23:773-9.

198 Duan FF, Liu JH, March JC. Engineered commensal bacteria reprogram intestinal cells into glucose-responsive insulin-secreting cells for the treatment of diabetes. Diabetes 2015;64:1794-803.

199 Kjems LL, Holst JJ, Vølund A, et al. The influence of GLP-1 on glucose-stimulated insulin secretion effects on $\beta$-cell sensitivity in type 2 and nondiabetic subjects. Diabetes 2003;52:380-6.

200 D'Alessio DA, Sandoval DA, Seeley RJ. New ways in which GLP-1 can regulate glucose homeostasis. J Clin Invest 2005;115:3406-8.

201 Knauf C, Cani PD, Kim DH, et al. Role of central nervous system glucagon-like peptide-1 receptors in enteric glucose sensing. Diabetes 2008;57:2603-12.

202 Sun F, Chai S, Li L, et al. Effects of glucagon-like peptide-1 receptor agonists on weight loss in patients with type 2 diabetes: a systematic review and network meta-analysis. J Diab Res 2015;2015:157201.

203 Bell GI, Sanchez-Pescador R, Laybourn PJ, et al. Exon duplication and divergence in the human preproglucagon gene. Nature 1983;304:368-71.

204 Orskov C, Rabenhøj L, Wettergren A, et al. Tissue and plasma concentrations of amidated and glycine-extended glucagon-like peptide I in humans. Diabetes 1994;43:535-9.

205 Duan F, Curtis KL, March JC. Secretion of insulinotropic proteins by commensal bacteria: rewiring the gut to treat diabetes. App/ Environ Microbiol 2008;74:7437-8.

206 Suzuki A, Nakauchi H, Taniguchi H. Glucagon-like peptide 1 (1-37) converts intestinal epithelial cells into insulin-producing cells. Proc Natl Acad Sci USA 2003;100:5034-9.

207 Bermúdez-Humarán LG, Kharrat P, Chatel J-M, et al. Lactococci and lactobacilli as mucosal delivery vectors for therapeutic proteins and DNA vaccines. Microb Cell Fact 2011;10(Suppl 1):S4.

208 Daniel C, Roussel Y, Kleerebezem M, et al. Recombinant lactic acid bacteria as mucosal biotherapeutic agents. Trends Biotechnol 2011;29:499-508.

209 Yadav H, Jain S, Sinha PR. Antidiabetic effect of probiotic dahi containing Lactobacillus acidophilus and Lactobacillus casei in high fructose fed rats. Nutrition 2007;23:62-8.

210 Yun SI, Park HO, Kang JH. Effect of Lactobacillus gasseri BNR17 on blood glucose levels and body weight in a mouse model of type 2 diabetes. J App/ Microbiol 2009;107:1681-6.

211 Ejtahed HS, Mohtadi-Nia J, Homayouni-Rad A, et al. Probiotic yogurt improves antioxidant status in type 2 diabetic patients. Nutrition 2012;28:539-43.

212 Asemi Z, Zare Z, Shakeri $\mathrm{H}$, et al. Effect of multispecies probiotic supplements on metabolic profiles, hs-CRP, and oxidative stress in patients with type 2 diabetes. Ann Nutr Metab 2013;63:1-9.

213 Cani PD, Geurts L, Matamoros S, et al. Glucose metabolism: focus on gut microbiota, the endocannabinoid system and beyond. Diabetes Metab 2014; 40:246-57.

214 Kimura I, Ozawa K, Inoue D, et al. The gut microbiota suppresses insulin-mediated fat accumulation via the short-chain fatty acid receptor GPR43. Nat Commun 2013:4:1829.

215 Tazoe H, Otomo Y, Kaji I, et al. Roles of short-chain fatty acids receptors, GPR41 and GPR43 on colonic functions. J Physiol Pharmacol 2008;59(Suppl 2):251-62.

216 Tappenden KA, Thomson AB, Wild GE, et al. Short-chain fatty acids increase proglucagon and ornithine decarboxylase messenger RNAs after intestinal resection in rats. JPEN J Parenter Enteral Nutr 1996;20:357-62.

217 Tolhurst G, Heffron H, Lam YS, et al. Short-chain fatty acids stimulate glucagon-like peptide-1 secretion via the G-protein-coupled receptor FFAR2. Diabetes 2012;61:364-71.

218 Nøhr MK, Pedersen MH, Gille A, et al. GPR41/FFAR3 and GPR43/FFAR2 as cosensors for short-chain fatty acids in enteroendocrine cells vs FFAR3 in enteric neurons and FFAR2 in enteric leukocytes. Endocrinology 2013; 154:3552-64.

219 Zaibi MS, Stocker CJ, O'Dowd J, et al. Roles of GPR41 and GPR43 in leptin secretory responses of murine adipocytes to short chain fatty acids. FEBS Lett 2010;584:2381-6.

220 Lin HV, Frassetto A, Kowalik EJ Jr, et al. Butyrate and propionate protect against diet-induced obesity and regulate gut hormones via free fatty acid receptor 3-independent mechanisms. PLoS ONE 2012;7:e35240.

221 Lovshin JA, Drucker DJ. Incretin-based therapies for type 2 diabetes mellitus. Nat Rev Endocrinol 2009:5:262-9.

222 Veldhorst MA, Westerterp KR, Westerterp-Plantenga MS. Gluconeogenesis and protein-induced satiety. Br J Nutr 2012;107:595-600.

223 Udayappan SD, Hartstra AV, Dallinga-Thie GM, et al. Intestinal microbiota and faecal transplantation as treatment modality for insulin resistance and type 2 diabetes mellitus. Clin Exp Immunol 2014;177:24-9.

224 Yadav $\mathrm{H}$, Lee JH, Lloyd J, et al. Beneficial metabolic effects of a probiotic via butyrate-induced GLP-1 hormone secretion. J Biol Chem 2013;288:25088-97. 


\section{Review}

225 Eloe-Fadrosh EA, Brady A, Crabtree J, et al. Functional dynamics of the gut microbiome in elderly people during probiotic consumption. MBio 2015;6:pii: e00231-15.

226 Troy-Fioramonti S, Demizieux L, Gresti J, et al. Acute activation of cannabinoid receptors by anandamide reduces gastro-intestinal motility and improves postprandial glycemia in mice. Diabetes 2015;64:808-18.

227 Muccioli GG, Naslain D, Bäckhed F, et al. The endocannabinoid system links gut microbiota to adipogenesis 2010 2010-01-01 00:00:00.

228 Syed SK, Bui HH, Beavers LS, et al. Regulation of GPR119 receptor activity with endocannabinoid-like lipids. Am J Physiol Endocrinol Metab 2012;303:E1469-78.

229 Ben-Shabat S, Fride E, Sheskin T, et al. An entourage effect: inactive endogenous fatty acid glycerol esters enhance 2-arachidonoyl-glycerol cannabinoid activity. Eur J Pharmacol 1998:353:23-31.

230 Taneera J, Jin Z, Jin Y, et al. gamma-Aminobutyric acid (GABA) signalling in human pancreatic islets is altered in type 2 diabetes. Diabetologia 2012;55:1985-94.
231 Tian J, Dang HN, Yong J, et al. Oral treatment with gamma-aminobutyric acid improves glucose tolerance and insulin sensitivity by inhibiting inflammation in high fat diet-fed mice. PLOS ONE 2011;6:e25338.

232 Alang N, Kelly CR. Weight gain after fecal microbiota transplantation. Open Forum Infect Dis 2015;2:ofv004.

\section{Answers}

1. True

2. False

3. True

4. False

5. False 